\title{
Concise Total Syntheses of Palominol, Dolabellatrienone, $\square$-Araneosene, and Isoedunol Via an Enantioselective Diels-Alder Macrobicyclization
}

\author{
Scott A. Snyder and E. J. Corey* \\ Department of Chemistry and Chemical Biology \\ Harvard University \\ Cambridge, Massachusetts 02138, USA
}

General Procedures. All reactions were carried out under an argon atmosphere with dry solvents under anhydrous conditions, unless otherwise noted. Dry tetrahydrofuran (THF), toluene, benzene, diethyl ether $\left(\mathrm{Et}_{2} \mathrm{O}\right)$, ethylene glycol dimethyl ether (DME), methylene chloride $\left(\mathrm{CH}_{2} \mathrm{Cl}_{2}\right)$, 1,2-dichloroethane, 1,8-diazabicyclo[5.4.0]undec-7-ene (DBU), methanol, triethylamine $\left(\mathrm{Et}_{3} \mathrm{~N}\right)$, and diisopropylethylamine $\left(i-\mathrm{Pr}_{2} \mathrm{NEt}\right)$ were obtained by drying over $\mathrm{Na}$ /benzophenone (THF, $\mathrm{Et}_{2} \mathrm{O}, \mathrm{DME}$ ) or $\mathrm{CaH}_{2}$ (toluene, benzene, $\mathrm{CH}_{2} \mathrm{Cl}_{2}$, 1,2-dichloroethane, DBU, methanol, $\mathrm{Et}_{3} \mathrm{~N}, i$ - $\left.\mathrm{Pr}_{2} \mathrm{NEt}\right)$. Yields refer to chromatographically and spectroscopically $\left({ }^{1} \mathrm{H}\right.$ and ${ }^{13} \mathrm{C}$ NMR) homogeneous materials, unless otherwise stated. Reagents were purchased at the highest commercial quality and used without further purification, unless otherwise stated. Reactions were monitored by thin-layer chromatography (TLC) carried out on $0.25 \mathrm{~mm}$ E. Merck silica gel plates (60F-254) using UV light as a visualizing agent and an ethanolic solution of phosphomolybdic acid/cerium sulfate, and heat as developing agents. E. Merck silica gel (60, particle size $0.040-0.063 \mathrm{~mm}$ ) was used for flash column chromatography. Preparative thinlayer chromatography separations were carried out on $0.50 \mathrm{~mm}$ E. Merck silica gel plates (60F254). NMR spectra were recorded on Bruker DRX-500, Varian Unity/INOVA 500, or Varian Mercury 400 instruments and calibrated using residual undeuterated solvent as an internal reference. The following abbreviations were used to explain the multiplicities: $\mathrm{s}=$ singlet, $\mathrm{d}=$ doublet, $\mathrm{t}=$ triplet, $\mathrm{q}=$ quartet, $\mathrm{m}=$ multiplet, quin $=$ quintuplet, sext $=$ sextet, sep $=$ septet, $\mathrm{br}=$ broad, app = apparent. IR spectra were recorded on an Applied Systems (ASI) ReactIR 1000 
spectrometer. Electrospray ionization (ESI) mass spectrometry (MS) experiments were performed on a Platform II mass spectrometer (Micromass, Inc., Beverly, MA). High-resolution mass spectra (HRMS) were recorded on an LCT mass spectrometer (Micromass, Inc., Beverly, MA) using a mass resolution of 5000 .

Abbreviations. TBDPS = tert-butyldiphenylsilyl, 4-DMAP = 4-dimethylaminopyridine, $\mathrm{Ac}=$ acetyl, NMP $=N$-methyl-2-pyrrolidinone, $\mathrm{dba}=$ trans, trans-dibenzylideneacetone, $\mathrm{TBAF}$ $=$ tetra- $n$-butylammonium fluoride, TIPS $=$ triisopropylsilyl, HPLC $=$ high-pressure liquid chromatography, NaHMDS $=$ sodium bis(trimethylsilyl)amide, IBX $=o$-iodoxybenzic acid, MPO = 4-methoxypyridine $N$-oxide, DMSO = dimethylsulfoxide, LDA = lithium diisopropylamide, TBS = tert-butyldimethylsilyl, MOM = methoxy methyl.

Vinyl stannane 6. ${ }^{1}$ Anhydrous $\mathrm{HBr}$ was bubbled into a suspension of $\mathrm{Et}_{4} \mathrm{NBr}(26.0 \mathrm{~g}$, $123.8 \mathrm{mmol}, 1.6$ equiv) in $\mathrm{CH}_{2} \mathrm{Cl}_{2}(90 \mathrm{~mL})$ at $0{ }^{\circ} \mathrm{C}$ until dissolution was complete, yielding a yellow solution. After warming the resultant reaction contents to $25^{\circ} \mathrm{C}$ and stirring for an additional $10 \mathrm{~min}$, propargyl alcohol $(4.16 \mathrm{~mL}, 77.4 \mathrm{mmol}, 1.0$ equiv) was added slowly from a syringe over $5 \mathrm{~min}$. The resultant mixture was stirred for $1 \mathrm{~h}$ at $25^{\circ} \mathrm{C}$, and then for $2 \mathrm{~h}$ at $45^{\circ} \mathrm{C}$. Upon completion, the reaction contents were cooled to $25^{\circ} \mathrm{C}$, diluted with $\mathrm{Et}_{2} \mathrm{O}(50 \mathrm{~mL})$, and filtered through a plug of silica gel using several $\mathrm{Et}_{2} \mathrm{O}$ washes to quantitate the transfer. The combined organic extracts were then dried $\left(\mathrm{MgSO}_{4}\right)$ and concentrated to yield 2-bromo-1propen-3-ol (10.5 g, 98\% yield) as a light yellow oil. Pressing forward without any additional purification, this newly-formed compound (10.5 g, $76.6 \mathrm{mmol}, 1.0$ equiv) was taken up in DMF $(250 \mathrm{~mL})$ and treated sequentially at $25^{\circ} \mathrm{C}$ with imidazole $(13.0 \mathrm{~g}, 191.6 \mathrm{mmol}, 2.5$ equiv $)$ and TBDPSCl $\left(25.1 \mathrm{~mL}, 95.8 \mathrm{mmol}, 1.25\right.$ equiv). After $12 \mathrm{~h}$ of stirring at $25^{\circ} \mathrm{C}$, the reaction mixture was poured into saturated aqueous $\mathrm{NaHCO}_{3}(300 \mathrm{~mL})$ and extracted with $\mathrm{Et}_{2} \mathrm{O}(3 \square 250$ $\mathrm{mL})$. The combined organic layers were then washed with $\mathrm{H}_{2} \mathrm{O}(100 \mathrm{~mL})$ and brine $(100 \mathrm{~mL})$, dried $\left(\mathrm{MgSO}_{4}\right)$, and concentrated. The resultant yellow residue was purified by flash column chromatography (silica gel, hexanes/EtOAc, 1:0 9:1) to give the desired TBDPS-protected vinyl bromide (22.4 g, 78\% yield) as a clear oil. $\mathrm{R}_{f}=0.60$ (silica gel, hexanes/EtOAc, 19:1); IR 
(film) $\square_{\max } 3072,3050,2961,2931,2858,1640,1473,1429,1113,1106,891,825,740,700$ $\mathrm{cm}^{-1}$; ${ }^{1} \mathrm{H}$ NMR (400 MHz, $\mathrm{CDCl}_{3}$ ) $\square 7.68-7.65$ (m, $\left.4 \mathrm{H}\right), 7.44-7.40$ (m, 6 H), 6.10 (m, $\left.1 \mathrm{H}\right), 5.58$ $(\mathrm{m}, 1 \mathrm{H}), 4.23$ (m, $1 \mathrm{H}), 1.08(\mathrm{~s}, 9 \mathrm{H}) ;{ }^{13} \mathrm{C} \mathrm{NMR}\left(100 \mathrm{MHz}, \mathrm{CDCl}_{3}\right) \square 135.7,133.1,131.4,130.1$, 128.1, 115.2, 68.1, 27.0, 19.5; HRMS (MALDI-FTMS) calcd for $\mathrm{C}_{19} \mathrm{H}_{27} \mathrm{NBrOSi}^{+}\left[\mathrm{M}+\mathrm{NH}_{4}^{+}\right]$ 392.1061, found 392.1046. A portion of this intermediate (4.40 g, $11.7 \mathrm{mmol}, 1.0$ equiv) was dissolved in benzene $(100 \mathrm{~mL})$ and treated sequentially at $25{ }^{\circ} \mathrm{C}$ with $i-\mathrm{PrNEt}_{2}(1.02 \mathrm{~mL}, 5.86$ mmol, 0.5 equiv), hexamethylditin (5.00 g, $15.3 \mathrm{mmol}, 1.3$ equiv), and $\mathrm{Pd}\left(\mathrm{PPh}_{3}\right)_{4}(1.32 \mathrm{~g}, 1.17$ mmol, 0.1 equiv). The resultant yellow-orange solution was degassed with bubbling argon for $30 \mathrm{~min}$, and the reaction contents were heated at $80{ }^{\circ} \mathrm{C}$ for $3 \mathrm{~h}$. Upon completion, the reaction mixture was cooled to $25^{\circ} \mathrm{C}$, poured into $\mathrm{H}_{2} \mathrm{O}(200 \mathrm{~mL})$, and extracted with EtOAc $(3 \square 150$ $\mathrm{mL})$. The combined organic layers were then washed with $\mathrm{H}_{2} \mathrm{O}(200 \mathrm{~mL})$ and brine $(200 \mathrm{~mL})$, dried $\left(\mathrm{MgSO}_{4}\right)$, and concentrated. The resultant yellow residue was purified by flash column chromatography (silica gel, hexanes/EtOAc, 1:0 19:1) to give vinyl stannane 6 (4.96 g, $92 \%$ yield) as a colorless oil. 6: $\mathrm{R}_{f}=0.67$ (silica gel, hexanes/EtOAc, 19:1); IR (film) $\square_{\max } 3071$, 3050, 2960, 2932, 2857, 1590, 1473, 1429, 1391, 1361, 1189, 1112, 1057, 998, 921, 829, 767, 741, $699 \mathrm{~cm}^{-1}$; ${ }^{1} \mathrm{H}$ NMR (400 MHz, $\left.\mathrm{CDCl}_{3}\right) \square 7.68$ (dd, $\left.J=7.6,1.6 \mathrm{~Hz}, 4 \mathrm{H}\right), 7.41$ (m, $\left.6 \mathrm{H}\right), 5.87$ $(\mathrm{d}, J=2.0 \mathrm{~Hz}, 1 \mathrm{H}), 5.25(\mathrm{~d}, J=2.0 \mathrm{~Hz}, 1 \mathrm{H}), 4.34(\mathrm{~d}, J=1.2 \mathrm{~Hz}, 2 \mathrm{H}), 1.06(\mathrm{~s}, 9 \mathrm{H}), 0.18(\mathrm{~s}, 9$ $\mathrm{H}) ;{ }^{13} \mathrm{C}$ NMR $\left(100 \mathrm{MHz}, \mathrm{CDCl}_{3}\right) \square 135.8,133.9,129.8,127.9,122.5,70.5,27.1,19.4,-9.1$; HRMS (MALDI-FTMS) calcd for $\mathrm{C}_{22} \mathrm{H}_{36} \mathrm{NOSiSn}^{+}\left[\mathrm{M}+\mathrm{NH}_{4}^{+}\right]$478.1588, found 478.1588.

Epoxy acetate 7. ${ }^{2}$ Trans,trans-farnesol (15.0 g, $67.5 \mathrm{mmol}, 1.0$ equiv) was dissolved in $\mathrm{CH}_{2} \mathrm{Cl}_{2}(250 \mathrm{~mL})$ and treated sequentially at $0{ }^{\circ} \mathrm{C}$ with $\mathrm{Et}_{3} \mathrm{~N}$ (14.1 mL, $102 \mathrm{mmol}, 1.5$ equiv), 4DMAP (0.07 g, catalytic), and $\mathrm{Ac}_{2} \mathrm{O}(7.0 \mathrm{~mL}, 74.2 \mathrm{mmol}, 1.2$ equiv). After $5 \mathrm{~min}$ of stirring at 0 ${ }^{\circ} \mathrm{C}$, the reaction mixture was poured into $\mathrm{H}_{2} \mathrm{O}(100 \mathrm{~mL})$ and extracted with $\mathrm{CH}_{2} \mathrm{Cl}_{2}(3 \square 50 \mathrm{~mL})$. The combined organic layers were then washed with brine $(100 \mathrm{~mL})$, dried $\left(\mathrm{MgSO}_{4}\right)$, and concentrated to give the desired acetylated product (17.8 g, 99\% yield) as a light yellow oil. Pressing forward without any additional purification, a portion of this intermediate $(8.80 \mathrm{~g}, 33.3$ mmol, 1.0 equiv) was dissolved in THF $(840 \mathrm{~mL})$, cooled to $0{ }^{\circ} \mathrm{C}$, and then diluted with $\mathrm{H}_{2} \mathrm{O}$ 
(640 mL) to give a cloudy solution. $N$-Bromosuccinimide (6.52 g, $36.6 \mathrm{mmol}, 1.1$ equiv) was then added portionwise over the course of $45 \mathrm{~min}$ at $0{ }^{\circ} \mathrm{C}$. Once the addition was complete, the suspension was stirred for $1 \mathrm{~h}$ at $0{ }^{\circ} \mathrm{C}$. The reaction contents were then concentrated with the internal temperature maintained at $5{ }^{\circ} \mathrm{C}$, taken up in $\operatorname{Et}_{2} \mathrm{O} /$ hexanes $(1: 1,200 \mathrm{~mL})$, and washed with $\mathrm{H}_{2} \mathrm{O}(3 \square 100 \mathrm{~mL})$. The organic layer was then dried $\left(\mathrm{MgSO}_{4}\right)$, concentrated, and purified by flash column chromatography (silica gel, hexanes/EtOAc, 1:0 $\square$ 4:1) to give the desired bromohydrin intermediate $(8.93 \mathrm{~g}, 71 \%$ yield) as a colorless oil. Next, the newly-formed bromohydrin (8.93 g, $23.5 \mathrm{mmol}, 1.0$ equiv) was taken up in $\mathrm{MeOH}(200 \mathrm{~mL})$ and treated with finely ground $\mathrm{K}_{2} \mathrm{CO}_{3}\left(8.11 \mathrm{~g}, 58.8 \mathrm{mmol}, 2.5\right.$ equiv) at $25^{\circ} \mathrm{C}$. Following $90 \mathrm{~min}$ of stirring at 25 ${ }^{\circ} \mathrm{C}$, the reaction contents were poured into $\mathrm{H}_{2} \mathrm{O}(200 \mathrm{~mL})$ and extracted with $\mathrm{Et}_{2} \mathrm{O}(3 \square 75 \mathrm{~mL})$. The combined organic layers were then washed with brine $(3 \square 75 \mathrm{~mL})$, dried $\left(\mathrm{MgSO}_{4}\right)$, and concentrated to give the desired epoxide (5.45 g, 97\% yield) as a colorless oil. Finally, this intermediate $\left(5.45 \mathrm{~g}, 22.9 \mathrm{mmol}, 1.0\right.$ equiv) was dissolved in $\mathrm{CH}_{2} \mathrm{Cl}_{2}$ (200 mL) and treated sequentially at $0{ }^{\circ} \mathrm{C}$ with $\mathrm{Et}_{3} \mathrm{~N}(4.78 \mathrm{~mL}, 34.3 \mathrm{mmol}, 1.5$ equiv), 4-DMAP (0.05 g, catalytic), and $\mathrm{Ac}_{2} \mathrm{O}\left(2.59 \mathrm{~mL}, 27.4 \mathrm{mmol}, 1.2\right.$ equiv). After $5 \mathrm{~min}$ of stirring at $0{ }^{\circ} \mathrm{C}$, the reaction mixture was poured into $\mathrm{H}_{2} \mathrm{O}(100 \mathrm{~mL})$ and extracted with $\mathrm{CH}_{2} \mathrm{Cl}_{2}(3 \square 75 \mathrm{~mL})$. The combined organic layers were then washed with brine $(100 \mathrm{~mL})$, dried $\left(\mathrm{MgSO}_{4}\right)$, and concentrated. The resultant yellow residue was purified by flash column chromatography (silica gel, hexanes/EtOAc, 1:0 5:1) to give epoxy acetate 7 (6.06 $\mathrm{g}, 95 \%$ yield) as a colorless oil. 7: $\mathrm{R}_{f}=0.41$ (silica gel, hexanes/EtOAc, 3:1); IR (film) $\square_{\max } 2927,1738,1447,1378,1366,1230,1117,1023,954,874$, $679 \mathrm{~cm}^{-1} ;{ }^{1} \mathrm{H}$ NMR $\left(400 \mathrm{MHz}, \mathrm{CDCl}_{3}\right) \square 5.33(\mathrm{~m}, 1 \mathrm{H}), 5.14(\mathrm{~m}, 1 \mathrm{H}), 4.57$ (d, J = $\left.7.2 \mathrm{~Hz}, 2 \mathrm{H}\right)$, $2.69(\mathrm{t}, J=6.4 \mathrm{~Hz}, 1 \mathrm{H}), 2.14-2.09(\mathrm{~m}, 4 \mathrm{H}), 2.08-2.03(\mathrm{~m}, 2 \mathrm{H}), 2.03(\mathrm{~s}, 3 \mathrm{H}), 1.69(\mathrm{~s}, 3 \mathrm{H})$, 1.68-1.66 (m, $2 \mathrm{H}), 1.59$ (s, $3 \mathrm{H}), 1.29(\mathrm{~s}, 3 \mathrm{H}), 1.25(\mathrm{~s}, 3 \mathrm{H}) ;{ }^{13} \mathrm{C} \mathrm{NMR}\left(100 \mathrm{MHz}, \mathrm{CDCl}_{3}\right) \square$ $171.4,142.3,134.8,124.5,118.6,64.4,61.6,58.5,39.6,36.5,27.7,26.4,25.1,21.3,19.0,16.7$, 16.2; HRMS (MALDI-FTMS) calcd for $\mathrm{C}_{17} \mathrm{H}_{29} \mathrm{O}_{3}{ }^{+}\left[\mathrm{M}+\mathrm{H}^{+}\right]$281.2116, found 281.2119.

†-Allyl Stille Coupling Product 8. A solution of epoxy acetate 7 (2.07 g, 7.39 mmol, 1.2 equiv) in 2-methyl- $N$-pyrrolidinone $(17 \mathrm{~mL})$ was added via cannula to a reaction vessel 
containing $\mathrm{Pd}_{2} \mathrm{dba}_{3}(0.677 \mathrm{~g}, 0.739 \mathrm{mmol}, 0.12$ equiv) and flame-dried $\mathrm{LiCl}$ (1.25 g, $29.6 \mathrm{mmol}$, 4.7 equiv) at $25^{\circ} \mathrm{C}$. A $3 \mathrm{~mL}$ wash of 2-methyl- $N$-pyrrolidinone was used to quantitate the transfer. A solution of stannane 6 (2.90 g, $6.31 \mathrm{mmol}, 1.0$ equiv) and $i$ - $\operatorname{Pr}_{2} \mathrm{NEt}(2.58 \mathrm{~mL}, 14.8$ mmol, 2.3 equiv) in 2-methyl- $N$-pyrrolidinone $(17 \mathrm{~mL})$ was then added via cannula, again using a $3 \mathrm{~mL}$ wash of 2-methyl- $N$-pyrrolidinone to quantitate the transfer. The resultant dark red solution was stirred at $25{ }^{\circ} \mathrm{C}$ for $10 \mathrm{~min}$, and then was warmed to $35^{\circ} \mathrm{C}$ and stirred for an additional $1.5 \mathrm{~h}$. Upon completion, the reaction contents were cooled to $25^{\circ} \mathrm{C}$, poured into saturated aqueous $\mathrm{NH}_{4} \mathrm{Cl}(100 \mathrm{~mL})$, and extracted with EtOAc $(3 \square 50 \mathrm{~mL})$. The combined organic layers were then washed with $\mathrm{H}_{2} \mathrm{O}(50 \mathrm{~mL})$ and brine $(50 \mathrm{~mL})$, dried $\left(\mathrm{MgSO}_{4}\right)$, and concentrated. The resultant red-brown residue was purified by flash column chromatography (silica gel, hexanes/EtOAc, 1:0 4:1) to give Stille coupling product 8 (3.12 g, 96\% yield) as a colorless oil. 8: $\mathrm{R}_{f}=0.56$ (silica gel, hexanes/EtOAc, 3:1); IR (film) $\square_{\max } 2960,2857,1473$, 1429, 1378, 1112, 898, 824, 741, $701 \mathrm{~cm}^{-1} ;{ }^{1} \mathrm{H}$ NMR (400 MHz, $\left.\mathrm{CDCl}_{3}\right) \square 7.74-7.71$ (m, $\left.4 \mathrm{H}\right)$, 7.48-7.42 (m, $6 \mathrm{H}), 5.19(\mathrm{~m}, 3 \mathrm{H}), 4.91(\mathrm{~s}, 1 \mathrm{H}), 4.14(\mathrm{~s}, 2 \mathrm{H}), 2.74(\mathrm{~m}, 3 \mathrm{H}), 2.18-2.04(\mathrm{~m}, 6 \mathrm{H})$, 1.70-1.68 (m, $1 \mathrm{H}), 1.65$ (s, $3 \mathrm{H}), 1.63$ (m, $1 \mathrm{H}), 1.61$ (s, $3 \mathrm{H}), 1.34$ (s, $3 \mathrm{H}), 1.29$ (s, $3 \mathrm{H}), 1.11$ (s, $9 \mathrm{H}) ;{ }^{13} \mathrm{C}$ NMR (100 MHz, $\left.\mathrm{CDCl}_{3}\right) \square 147.8,137.0,135.8,134.4,133.9,129.8,127.9,125.0$, $121.5,108.8,66.7,64.4,58.5,39.9,36.5,31.6,27.7,27.0,26.8,25.1,19.5,19.0,16.2,16.1$; HRMS (MALDI-FTMS) calcd for $\mathrm{C}_{34} \mathrm{H}_{49} \mathrm{O}_{2} \mathrm{Si}^{+}\left[\mathrm{M}+\mathrm{H}^{+}\right]$517.3502, found 517.3508.

Horner-Wadsworth-Emmons Adduct 10. Intermediate 8 (2.49 g, $4.82 \mathrm{mmol}, 1.0$ equiv) was taken up in $\mathrm{THF} / \mathrm{H}_{2} \mathrm{O}(10: 1,55 \mathrm{~mL})$ and treated sequentially at $0{ }^{\circ} \mathrm{C}$ with $\mathrm{NaIO}_{4}$ (0.618 g, $2.89 \mathrm{mmol}, 0.6$ equiv) and $\mathrm{HIO}_{4} \cdot 2 \mathrm{H}_{2} \mathrm{O}(1.21 \mathrm{~g}, 5.30 \mathrm{mmol}, 1.1$ equiv). The resultant biphasic mixture was stirred at $0{ }^{\circ} \mathrm{C}$ for $10 \mathrm{~min}$ and then warmed to $25^{\circ} \mathrm{C}$. After $45 \mathrm{~min}$, the reaction contents were quenched by the addition of saturated aqueous $\mathrm{NaHCO}_{3}(25 \mathrm{~mL})$, poured into $\mathrm{H}_{2} \mathrm{O}(25 \mathrm{~mL})$, and extracted with EtOAc $(3 \square 25 \mathrm{~mL})$. The combined organic layers were then washed with brine $(25 \mathrm{~mL})$, dried $\left(\mathrm{MgSO}_{4}\right)$, and concentrated to give the desired intermediate aldehyde $(2.25 \mathrm{~g}, 98 \%$ yield $)$ as a colorless oil. Next, diethyl-2-oxopropyl phosphonate (9, $2.76 \mathrm{~g}, 14.2 \mathrm{mmol}, 3.0$ equiv) was added to a suspension of $\mathrm{KO} t$ - $\mathrm{Bu}(1.33 \mathrm{~g}$, 
$11.8 \mathrm{mmol}, 2.5$ equiv) in $\mathrm{THF}(40 \mathrm{~mL})$ at $-78{ }^{\circ} \mathrm{C}$. After stirring the resultant solution for $30 \mathrm{~min}$ at $-78{ }^{\circ} \mathrm{C}$, a solution of the crude aldehyde (2.25 g, $4.73 \mathrm{mmol}, 1.0$ equiv) in THF (10 mL) was added dropwise over $5 \mathrm{~min}$. The reaction solution was then stirred for $20 \mathrm{~min}$ at $-78{ }^{\circ} \mathrm{C}$ and slowly warmed to $-20^{\circ} \mathrm{C}$ over the course of $2 \mathrm{~h}$. Upon completion, the reaction contents were quenched by the addition of saturated aqueous $\mathrm{NH}_{4} \mathrm{Cl}(25 \mathrm{~mL})$, poured into $\mathrm{H}_{2} \mathrm{O}(50 \mathrm{~mL})$, and extracted with EtOAc $(3 \square 25 \mathrm{~mL})$. The combined organic layers were then dried $\left(\mathrm{MgSO}_{4}\right)$, concentrated, and purified by flash column chromatography (silica gel, hexanes/EtOAc, 1:0 4:1) to give methyl ketone $\mathbf{1 0}\left(2.29 \mathrm{~g}, 94 \%\right.$ yield) as a colorless oil. 10: $\mathrm{R}_{f}=0.49$ (silica gel, hexanes/EtOAc, 3:1); IR (film) $\square_{\max } 2932,2857,1677,1627,1429,1361,1254,1112,897$, 825, 742, $702 \mathrm{~cm}^{-1}$; ${ }^{1} \mathrm{H}$ NMR (400 MHz, $\mathrm{CDCl}_{3}$ ) $\square 7.74-7.71(\mathrm{~m}, 4 \mathrm{H}), 7.48-7.40$ (m, $\left.6 \mathrm{H}\right), 6.80$ $(\mathrm{dt}, J=16.0,6.4 \mathrm{~Hz}, 1 \mathrm{H}), 6.10(\mathrm{~d}, J=16.0 \mathrm{~Hz}, 1 \mathrm{H}), 5.18(\mathrm{~m}, 3 \mathrm{H}), 4.90(\mathrm{~s}, 1 \mathrm{H}), 4.14(\mathrm{~s}, 2 \mathrm{H})$, $2.73(\mathrm{~d}, J=7.2 \mathrm{~Hz}, 2 \mathrm{H}), 2.35(\mathrm{t}, J=7.2 \mathrm{~Hz}, 2 \mathrm{H}), 2.27(\mathrm{~s}, 3 \mathrm{H}), 2.16(\mathrm{t}, J=7.6 \mathrm{~Hz}, 2 \mathrm{H}), 2.10(\mathrm{t}$, $J=7.2 \mathrm{~Hz}, 2 \mathrm{H}), 2.03(\mathrm{~m}, 2 \mathrm{H}), 1.64(\mathrm{~s}, 3 \mathrm{H}), 1.61(\mathrm{~s}, 3 \mathrm{H}), 1.10(\mathrm{~s}, 9 \mathrm{H}) ;{ }^{13} \mathrm{C} \mathrm{NMR}(100 \mathrm{MHz}$, $\left.\mathrm{CDCl}_{3}\right) \square 198.7,148.2,147.6,136.7,135.6,133.7,133.5,131.4,129.6,127.7,125.4,121.4$, 108.7, 66.5, 39.6, 38.0, 31.4, 31.0, 26.8 (2 C), 26.5, 26.4, 19.3, 16.0; HRMS (MALDI-FTMS) calcd for $\mathrm{C}_{34} \mathrm{H}_{47} \mathrm{O}_{2} \mathrm{Si}^{+}\left[\mathrm{M}+\mathrm{H}^{+}\right]$515.3345, found 515.3334.

TIPS enol ether 11. Methyl ketone 10 (9.15 g, $17.8 \mathrm{mmol}, 1.0$ equiv) was dissolved in THF $(150 \mathrm{~mL})$ and treated sequentially at $0{ }^{\circ} \mathrm{C}$ with $\mathrm{AcOH}(5 \mathrm{~mL})$ and TBAF $(44.4 \mathrm{~mL}, 1.0 \mathrm{M}$ in THF, 44.4 mmol, 42.5 equiv). The resultant yellow mixture was then warmed to $25{ }^{\circ} \mathrm{C}$ and stirred for $24 \mathrm{~h}$. Upon completion, the reaction contents were poured into saturated aqueous $\mathrm{NaHCO}_{3}(250 \mathrm{~mL})$ and extracted with EtOAc $(3 \square 100 \mathrm{~mL})$. The combined organic layers were then washed with $\mathrm{H}_{2} \mathrm{O}(75 \mathrm{~mL})$ and brine $(75 \mathrm{~mL})$, dried $\left(\mathrm{MgSO}_{4}\right)$, and concentrated. The resultant yellow residue was purified by flash column chromatography (silica gel, hexanes/EtOAc, 1:0 1:1) to give the desired alcohol (4.61 g, 94\% yield) as a colorless oil. $\mathrm{R}_{f}=$ 0.14 (silica gel, hexanes/EtOAc, 3:1); IR (film) $\square_{\max }$ 3430, 2914, 2854, 1673, 1625, 1432, 1360, 1254, 1057, 1025, 978, $897 \mathrm{~cm}^{-1} ;{ }^{1} \mathrm{H}$ NMR (400 MHz, $\left.\mathrm{CDCl}_{3}\right) \square 6.75(\mathrm{dt}, J=16.0,7.2 \mathrm{~Hz}, 1 \mathrm{H})$, $6.03(\mathrm{~d}, J=16.0 \mathrm{~Hz}, 1 \mathrm{H}), 5.15(\mathrm{t}, J=7.2 \mathrm{~Hz}, 1 \mathrm{H}), 5.11(\mathrm{t}, J=8.0 \mathrm{~Hz}, 1 \mathrm{H}), 4.98(\mathrm{~s}, 1 \mathrm{H}), 4.84$ 
(s, $1 \mathrm{H}), 4.03$ (s, $2 \mathrm{H}), 2.73$ (d, J = 7.2 Hz, $2 \mathrm{H}), 2.29$ (app q, $J=7.2 \mathrm{~Hz}, 2 \mathrm{H}), 2.20$ (s, $3 \mathrm{H}), 2.08$ (m, $4 \mathrm{H}), 2.00$ (m, $3 \mathrm{H}), 1.59$ (s, $3 \mathrm{H}), 1.58$ (s, $3 \mathrm{H}) ;{ }^{13} \mathrm{C}$ NMR (100 MHz, $\left.\mathrm{CDCl}_{3}\right) \square 198.8,148.3$ $(\mathrm{t}, J=36.4 \mathrm{~Hz}), 148.2,136.8,133.4,131.2,125.1(\mathrm{~d}, J=36.4 \mathrm{~Hz}), 121.2(\mathrm{~d}, J=36.4 \mathrm{~Hz}), 109.2$ $(\mathrm{t}, J=36.4 \mathrm{~Hz}), 65.7,39.5,37.9,31.6,30.8,26.6,26.3,15.8(\mathrm{t}, J=15.2 \mathrm{~Hz})$; HRMS (MALDIFTMS) calcd for $\mathrm{C}_{18} \mathrm{H}_{29} \mathrm{O}_{2}^{+}\left[\mathrm{M}+\mathrm{H}^{+}\right]$277.2167, found 277.2161. A portion of this alcohol (1.33 $\mathrm{g}, 4.81 \mathrm{mmol}, 1.0$ equiv) was dissolved in $\mathrm{CH}_{2} \mathrm{Cl}_{2}(60 \mathrm{~mL})$ and treated sequentially at $0{ }^{\circ} \mathrm{C}$ with solid $\mathrm{NaHCO}_{3}$ (4.04 g, $48.1 \mathrm{mmol}, 10$ equiv) and Dess-Martin periodinane ${ }^{3}$ (4.08 g, $9.62 \mathrm{mmol}$, 2.0 equiv). Following 90 min of stirring at $0{ }^{\circ} \mathrm{C}$, the reaction contents were poured into saturated aqueous $\mathrm{NaHCO}_{3}(25 \mathrm{~mL})$ and extracted with $\mathrm{CH}_{2} \mathrm{Cl}_{2}(3 \square 25 \mathrm{~mL})$. The combined organic layers were then washed with saturated aqueous $\mathrm{Na}_{2} \mathrm{SO}_{3}(3 \square 25 \mathrm{~mL})$, dried $\left(\mathrm{MgSO}_{4}\right)$, and concentrated. The resultant yellow residue was purified by flash column chromatography (silica gel, hexanes/EtOAc, 1:0 3:1) to give the expected aldehyde (1.23 g, 93\% yield) as a colorless oil. Alternatively, the alcohol $\left(3.65 \mathrm{~g}, 13.2 \mathrm{mmol}, 1.0\right.$ equiv) was taken up in $\mathrm{CH}_{2} \mathrm{Cl}_{2}(125 \mathrm{~mL})$ and treated with $\mathrm{MnO}_{2}(11.1 \mathrm{~g}, 132 \mathrm{mmol}, 10$ equiv $)$ at $25^{\circ} \mathrm{C}$. Following $24 \mathrm{~h}$ of stirring at $25^{\circ} \mathrm{C}$, the reaction contents were filtered through Celite using several $\mathrm{CH}_{2} \mathrm{Cl}_{2}$ washes to quantitate the transfer. The filtrate was then concentrated to give the desired aldehyde $(3.41 \mathrm{~g}, 94 \%$ yield $)$ as a colorless oil. $\mathrm{R}_{f}=0.31$ (silica gel, hexanes/EtOAc, 3:1); IR (film) $\square_{\max } 2915,2852,1692,1675$, 1626, 1432, 1360, 1254, 960, 874, $805 \mathrm{~cm}^{-1}$; ${ }^{1} \mathrm{H}$ NMR (400 MHz, $\left.\mathrm{CDCl}_{3}\right) \square 9.61(\mathrm{~s}, 1 \mathrm{H}), 6.80$ $(\mathrm{dt}, J=16.0,7.2 \mathrm{~Hz}, 1 \mathrm{H}), 6.24(\mathrm{~s}, 1 \mathrm{H}), 6.09(\mathrm{~d}, J=16.0 \mathrm{~Hz}, 1 \mathrm{H}), 6.02(\mathrm{~s}, 1 \mathrm{H}), 5.19(\mathrm{t}, J=7.2$ $\mathrm{Hz}, 1 \mathrm{H}), 5.17(\mathrm{t}, J=7.2 \mathrm{~Hz}, 1 \mathrm{H}), 2.95(\operatorname{app~d}, J=6.8 \mathrm{~Hz}, 2 \mathrm{H}), 2.35(\mathrm{~m}, 2 \mathrm{H}), 2.26(\mathrm{~s}, 3 \mathrm{H})$, 2.18-2.11 (m, $6 \mathrm{H}), 2.07(\mathrm{~m}, 2 \mathrm{H}), 1.64(\operatorname{app~s,} 6 \mathrm{H}) ;{ }^{13} \mathrm{C} \mathrm{NMR}\left(100 \mathrm{MHz}, \mathrm{CDCl}_{3}\right) \square 198.7$, $194.6,149.3,148.1,138.0,133.9,133.7,131.4,125.2,119.6,39.6,38.0,31.0,26.8,26.5,26.3$, 16.0 (2 C); HRMS (MALDI-FTMS) calcd for $\mathrm{C}_{18} \mathrm{H}_{27} \mathrm{O}_{2}^{+}\left[\mathrm{M}+\mathrm{H}^{+}\right]$275.2011, found 275.2011. Finally, the intermediate aldehyde $\left(0.500 \mathrm{~g}, 1.82 \mathrm{mmol}, 1.0\right.$ equiv) was dissolved in $\mathrm{CH}_{2} \mathrm{Cl}_{2}$, cooled to $-78{ }^{\circ} \mathrm{C}$, and treated sequentially with $i-\operatorname{Pr}_{2} \mathrm{NEt}(1.27 \mathrm{~mL}, 7.29 \mathrm{mmol}, 4.0$ equiv) and TIPSOTf $\left(0.539 \mathrm{~mL}, 2.00 \mathrm{mmol}, 1.1\right.$ equiv). After exactly $3 \mathrm{~min}$ of stirring at $-78^{\circ} \mathrm{C}$, the reaction contents were quenched by the rapid sequential addition of $\mathrm{Et}_{3} \mathrm{~N}(0.5 \mathrm{~mL})$ and $\mathrm{MeOH}$ 
$(0.5 \mathrm{~mL})$, stirred for an additional $10 \mathrm{~min}$ at $-78{ }^{\circ} \mathrm{C}$, warmed to $25{ }^{\circ} \mathrm{C}$, and then poured into saturated aqueous $\mathrm{NaHCO}_{3}(25 \mathrm{~mL})$. The aqueous phase was extracted with $\mathrm{CH}_{2} \mathrm{Cl}_{2}(3 \square 25 \mathrm{~mL})$ and the combined organic layers were then dried $\left(\mathrm{Na}_{2} \mathrm{SO}_{4}\right)$ and concentrated. The resultant pale yellow residue was purified by flash column chromatography $\left(\mathrm{Et}_{3} \mathrm{~N}\right.$-deactivated silica gel, hexanes/EtOAc, 1:0 4:1) to give TIPS enol ether 11 (0.651 g, 83\% yield) as a colorless oil along with a portion of recovered methyl ketone $(0.031 \mathrm{~g}, 88 \%$ yield based on recovered starting material). 11: $\mathrm{R}_{f}=0.49$ (silica gel, hexanes/EtOAc, 9:1); IR (film) $\square_{\max } 2944,2867,1696,1590$, $1465,1370,1239,1025,961,884,809,731,685 \mathrm{~cm}^{-1} ;{ }^{1} \mathrm{H}$ NMR (400 MHz, $\left.\mathrm{CDCl}_{3}\right) \square 9.59(\mathrm{~s}, 1$ H), $6.22(\mathrm{~s}, 1 \mathrm{H}), 6.04(\mathrm{dt}, J=15.6,6.8 \mathrm{~Hz}, 1 \mathrm{H}), 5.99(\mathrm{~s}, 1 \mathrm{H}), 5.86(\mathrm{~d}, J=16.0 \mathrm{~Hz}, 1 \mathrm{H}), 5.17$ $(\mathrm{t}, J=7.2 \mathrm{~Hz}, 1 \mathrm{H}), 5.12(\mathrm{t}, J=6.8 \mathrm{~Hz}, 1 \mathrm{H}), 2.93(\operatorname{app~d}, J=7.2 \mathrm{~Hz}, 2 \mathrm{H}), 2.19(\mathrm{~m}, 2 \mathrm{H})$, 2.10-2.05 (m, $6 \mathrm{H}), 1.61(\mathrm{~s}, 3 \mathrm{H}), 1.60(\mathrm{~s}, 3 \mathrm{H}), 1.24(\mathrm{~m}, 3 \mathrm{H}), 1.11(\mathrm{~s}, 9 \mathrm{H}), 1.09(\mathrm{~s}, 9 \mathrm{H}) ;{ }^{13} \mathrm{C}$ NMR (100 MHz, $\left.\mathrm{CDCl}_{3}\right) \square 194.8,155.6,149.5,138.5,134.8,134.1,131.4,128.1,124.7,119.6$, 93.2, 39.9, 39.5, 30.9, 26.7, 26.4, 18.3, 18.2, 16.2, 13.0; HRMS (MALDI-FTMS) calcd for $\mathrm{C}_{27} \mathrm{H}_{47} \mathrm{O}_{2} \mathrm{Si}^{+}\left[\mathrm{M}+\mathrm{H}^{+}\right]$431.3345, found 431.3338.

Oxazaborolidinium catalyst $(S)-4$. A $25-\mathrm{mL}$, round-bottom flask equipped with a stirring bar and a Dean-Stark trap (filled with $4 \AA$ molecular sieves, fitted with a reflux condenser, and equipped with a Teflon stopcock) was charged with (S)-(-)- $\square, \square-$ diphenyl-2pyrrolidinemethanol $\left(0.120 \mathrm{~g}, 0.474 \mathrm{mmol}, 1.0\right.$ equiv), tri-o-tolylboroxine ${ }^{4}(0.055 \mathrm{~g}, 0.156$ mmol, 0.33 equiv), and toluene $(20 \mathrm{~mL})$. The resulting solution was heated at reflux for $12 \mathrm{~h}$ using an oil bath maintained at a temperature of approximately $145^{\circ} \mathrm{C}$. Upon completion, the solvent was drained from the Dean-Stark trap and the contents of the reaction flask were reduced to a volume of $\sim 2 \mathrm{~mL}$ through distillation; the Dean-Stark trap was then closed, recharged with toluene $(20 \mathrm{~mL})$, and the same distillation procedure was repeated. After three iterations of this azeotroping process, the reaction solution (approximate volume of $2 \mathrm{~mL}$ ) was cooled to $25^{\circ} \mathrm{C}$, and the Dean-Stark trap was quickly replaced with a rubber septum and nitrogen inlet adaptor. The reaction contents were then cooled to $-20{ }^{\circ} \mathrm{C}$ and a solution of trifluoromethanesulfonimide $(0.093 \mathrm{~g}, 0.332 \mathrm{mmol}, 0.7 \mathrm{equiv})$ in toluene $(1 \mathrm{~mL})$ was added dropwise over the course of 2 
min. After 20 min of additional stirring at $-20^{\circ} \mathrm{C}$, a colorless, homogeneous solution of the cationic catalyst $(S)-4(0.332 \mathrm{mmol})$ was obtained.

Diels-Alder macrocycle 12. To a solution of freshly prepared catalyst $(S)-4(0.332$ mmol, 0.2 equiv) in toluene $(3 \mathrm{~mL})$ at $-93{ }^{\circ} \mathrm{C}$ was slowly added a solution of TIPS-enol ether 11 ( $0.732 \mathrm{~g}, 1.70 \mathrm{mmol}, 1.0$ equiv) as a solution in toluene $(2 \mathrm{~mL})$ over the course of $15 \mathrm{~min}$. The resultant yellow solution was stirred for $3 \mathrm{~h}$ at $-93{ }^{\circ} \mathrm{C}$, allowed to warm slowly to $-78{ }^{\circ} \mathrm{C}$, and stirred for an additional $10 \mathrm{~h}$ at $-78{ }^{\circ} \mathrm{C}$. Upon completion, the reaction contents were quenched by the addition of $\mathrm{Et}_{3} \mathrm{~N}(0.5 \mathrm{~mL})$, warmed to $25^{\circ} \mathrm{C}$, concentrated, and purified directly by flash column chromatography $\left(\mathrm{Et}_{3} \mathrm{~N}\right.$-deactivated silica gel, hexanes/EtOAc, 1:0 9:1) to give Diels-Alder macrocycle $12\left(0.524 \mathrm{~g}, 72 \%\right.$ yield) as a colorless oil. 12: $\mathrm{R}_{f}=0.53$ (silica gel, hexanes/EtOAc, 9:1); [ []$_{\mathrm{D}}{ }^{25}=-56.5^{\circ}\left(c=0.40, \mathrm{CHCl}_{3}, 90 \%\right.$ e.e. $)$; IR (film) $\square_{\max } 2943,2865$, $1719,1671,1463,1372,1204,1189,1055,1015,882,685 \mathrm{~cm}^{-1} ;{ }^{1} \mathrm{H}$ NMR $\left(400 \mathrm{MHz}, \mathrm{CDCl}_{3}\right) \square$ $9.81(\mathrm{~s}, 1 \mathrm{H}), 4.99(\mathrm{t}, J=6.0 \mathrm{~Hz}, 1 \mathrm{H}), 4.87(\mathrm{~m}, 2 \mathrm{H}), 2.51(\mathrm{~m}, 1 \mathrm{H}), 2.41(\mathrm{dd}, J=15.2,8.4 \mathrm{~Hz}, 1$ H), 2.30-2.09 (m, 8 H), 1.97 (dd, $J=15.2,4.8 \mathrm{~Hz}, 1 \mathrm{H}), 1.90-1.84$ (m, $3 \mathrm{H}), 1.60$ (s, $3 \mathrm{H}), 1.52$ (s, $3 \mathrm{H}), 1.43$ (m, $1 \mathrm{H}), 1.19-1.10$ (m, $21 \mathrm{H}) ;{ }^{13} \mathrm{C}$ NMR (100 MHz, CDCl $\left.{ }_{3}\right) \square 207.4,149.8,136.7$, $135.6,126.2$, 122.2, 107.4, 51.6, 39.6, 37.3, 35.5, 31.6, 30.6, 30.2, 27.0, 25.2, 18.2, 17.5, 16.0, 12.8; HRMS (MALDI-FTMS) calcd for $\mathrm{C}_{27} \mathrm{H}_{47} \mathrm{O}_{2} \mathrm{Si}^{+}\left[\mathrm{M}+\mathrm{H}^{+}\right] 431.3345$, found 431.3339. The enantioselectivity was determined by HPLC analysis using a Regis Whelk-O column $(25 \mathrm{~cm} \square$ $4.6 \mathrm{~mm}$ ) eluting with hexanes/ $i-\mathrm{PrOH}(99.9: 0.1)$ at a flow rate of $1.0 \mathrm{~mL} / \mathrm{min}$ and measuring UV absorbance at $215 \mathrm{~nm}$; retention times: $28.0 \mathrm{~min}$ (major) and $31.5 \mathrm{~min}$ (minor). The absolute configuration was determined by X-ray crystallographic analysis of derivative S1 prepared from 12 as described below.

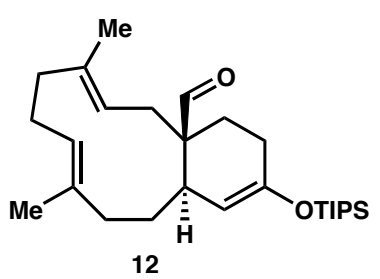

12

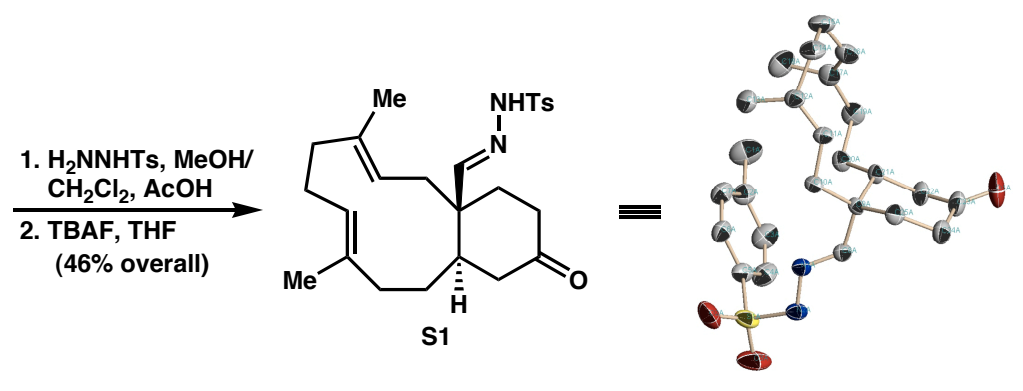


Crystalline derivative S1. To a solution of $12(0.041 \mathrm{~g}, 0.095 \mathrm{mmol}, 1.0$ equiv) in $\mathrm{MeOH} / \mathrm{CH}_{2} \mathrm{Cl}_{2}(5: 1,1.2 \mathrm{~mL})$ at $25{ }^{\circ} \mathrm{C}$ was added sequentially $p$-toluenesulfonhydrazide $(0.027 \mathrm{~g}$, $0.143 \mathrm{mmol}, 1.5$ equiv) and $\mathrm{AcOH}$ ( 2 drops). After stirring the resultant solution for $4 \mathrm{~h}$ at 25 ${ }^{\circ} \mathrm{C}$, the reaction mixture was concentrated directly and purified by flash column chromatography (silica gel, hexanes/EtOAc, 1:0\ 4:1) to give the desired hydrazone intermediate (0.038 g, 67\% yield) as a white foam. This compound $(0.038 \mathrm{~g}, 0.063 \mathrm{mmol}, 1.0$ equiv) was then taken up in THF $(1 \mathrm{~mL})$ and treated at $25^{\circ} \mathrm{C}$ with TBAF $(0.076 \mathrm{~mL}, 1.0 \mathrm{M}$ in THF, $0.076 \mathrm{mmol}, 1.2$ equiv). After 5 min of stirring at $25^{\circ} \mathrm{C}$, the reaction mixture was poured into saturated aqueous $\mathrm{NH}_{4} \mathrm{Cl}$ $(25 \mathrm{~mL})$ and extracted with $\mathrm{CH}_{2} \mathrm{Cl}_{2}(3 \square 25 \mathrm{~mL})$. The combined organic layers were then washed with $\mathrm{H}_{2} \mathrm{O}(25 \mathrm{~mL})$ and brine $(25 \mathrm{~mL})$, dried $\left(\mathrm{MgSO}_{4}\right)$, and concentrated. The resultant yellow residue was purified by flash column chromatography (silica gel, hexanes/EtOAc, 1:0 1:1) to give derivative $\mathbf{S 1}$ (0.019 g, 68\% yield) as a white solid which crystallized from hexanes/ $/ \mathrm{Et}_{2} \mathrm{O} / \mathrm{CH}_{2} \mathrm{Cl}_{2}$ (3:1:1). S1: $\mathrm{R}_{f}=0.29$ (silica gel, hexanes/EtOAc, 1:1); m.p. $=68.5-70.0$ ${ }^{\circ} \mathrm{C} ;[\square]_{\mathrm{D}}^{25}=-7.1^{\circ}\left(c=0.45, \mathrm{CHCl}_{3}, 90 \%\right.$ e.e. $)$; IR (film) $\square_{\max } 3184,2981,2917,1708,1451$, 1368, 1328, 1187, 1165, 1032, 909, 814, $727 \mathrm{~cm}^{-1}$; ${ }^{1} \mathrm{H}$ NMR (400 MHz, CDCl $) \square 8.02(\mathrm{~s}, 1 \mathrm{H})$, $7.80(\mathrm{~d}, J=8.0 \mathrm{~Hz}, 2 \mathrm{H}), 7.30(\mathrm{~d}, J=8.0 \mathrm{~Hz}, 2 \mathrm{H}), 7.17(\mathrm{~s}, 1 \mathrm{H}), 4.71(\mathrm{~m}, 2 \mathrm{H}), 2.41(\mathrm{~s}, 3 \mathrm{H})$, $2.33(\mathrm{dd}, J=15.6,9.2 \mathrm{~Hz}, 1 \mathrm{H}), 2.29(\mathrm{~d}, J=4.4 \mathrm{~Hz}, 1 \mathrm{H}), 2.26(\mathrm{~d}, J=4.0 \mathrm{~Hz}, 1 \mathrm{H}), 2.22(\mathrm{~m}, 1$ H), 2.15-2.10 (m, 2 H), 2.08-2.00 (m, 2 H), 1.98 (app t, $J=5.2 \mathrm{~Hz}, 1 \mathrm{H}), 1.95-1.81$ (m, $6 \mathrm{H})$, 1.57 (app t, $J=10.8 \mathrm{~Hz}, 1 \mathrm{H}), 1.50(\mathrm{~s}, 3 \mathrm{H}), 1.44(\mathrm{~s}, 3 \mathrm{H}), 1.09$ (app dd, $J=9.6,6.0 \mathrm{~Hz}, 1 \mathrm{H}) ;{ }^{13} \mathrm{C}$ NMR $\left(100 \mathrm{MHz}, \mathrm{CDCl}_{3}\right) \square 211.5,154.7,144.6,136.5,136.4,134.8,129.7,128.0,124.7,120.5$, 44.3, 44.0, 40.3, 39.2, 37.7, 36.3, 34.0, 32.8, 30.6, 24.8, 21.6, 17.7, 15.9; HRMS (MALDIFTMS) calcd for $\mathrm{C}_{25} \mathrm{H}_{35} \mathrm{~N}_{2} \mathrm{O}_{3} \mathrm{~S}^{+}\left[\mathrm{M}+\mathrm{H}^{+}\right] 443.2368$, found 443.2370. 
Table S1. Optimization of the enantioselectivity of the Diels-Alder reaction converting 11 into 12.

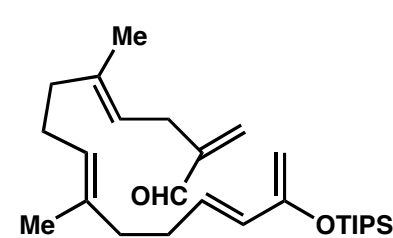

11

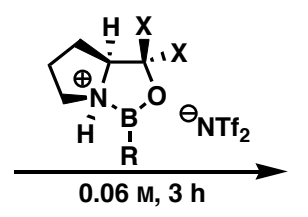

$0.06 \mathrm{M}, 3 \mathrm{~h}$

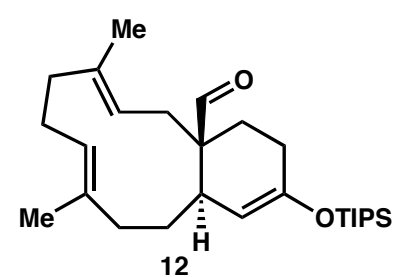

12

\begin{tabular}{c|ccccccc} 
Entry & Catalyst & Solvent & Temp & $\mathrm{X}$ & $\mathrm{R}$ & endo/exo & e.e. \\
\hline 1 & $(S)$ & toluene & $-78{ }^{\circ} \mathrm{C}$ & $\mathrm{Ph}$ & $2-\mathrm{MePh}$ & $20: 1$ & 80.3 \\
2 & $(R)$ & toluene & $-78{ }^{\circ} \mathrm{C}$ & $\mathrm{Ph}$ & $2-\mathrm{MePh}$ & $20: 1$ & 86.4 \\
3 & $(S)$ & $\mathrm{CH}_{2} \mathrm{Cl}_{2}$ & $-78{ }^{\circ} \mathrm{C}$ & $\mathrm{Ph}$ & $2-\mathrm{MePh}$ & $20: 1$ & 55.8 \\
4 & $(S)$ & toluene & $-78{ }^{\circ} \mathrm{C}$ & $\mathrm{Ph}$ & $\mathrm{Ph}$ & $13: 1$ & 87.2 \\
5 & $(S)$ & $\mathrm{CH}_{2} \mathrm{Cl}_{2}$ & $-78{ }^{\circ} \mathrm{C}$ & $\mathrm{Ph}$ & $\mathrm{Ph}$ & $12: 1$ & 69.6 \\
6 & $(S)$ & toluene & $-93^{\circ} \mathrm{C}$ & $\mathrm{Mes}$ & $\mathrm{Ph}$ & $12: 1$ & 87.8 \\
7 & $(S)$ & toluene & $-93^{\circ} \mathrm{C}$ & $\mathrm{Mes}$ & $2-\mathrm{MePh}$ & $12: 1$ & 87.7 \\
8 & $(S)$ & toluene & $-93^{\circ} \mathrm{C}$ & $\mathrm{Ph}$ & $2-\mathrm{MePh}$ & $20: 1$ & 90.4
\end{tabular}

$\mathrm{Me}_{2} \mathrm{AlCH}_{2} \mathrm{CH}_{2} \mathrm{AlMe}_{2}{ }^{{ }^{5}} \mathrm{Me}_{3} \mathrm{Al}(1.00 \mathrm{~mL}, 2.0 \mathrm{M}$ in toluene, $2.00 \mathrm{mmol}, 2.0$ equiv) was dissolved in $\mathrm{CH}_{2} \mathrm{Cl}_{2}(2 \mathrm{~mL})$, cooled to $-78^{\circ} \mathrm{C}$, and treated slowly with 1,2-ethanedithiol (0.084 $\mathrm{mL}, 1.00 \mathrm{mmol}, 1.0$ equiv). After $5 \mathrm{~min}$ of stirring at $-78{ }^{\circ} \mathrm{C}$, the resultant white slurry was warmed to $0{ }^{\circ} \mathrm{C}$ and stirred for an additional $30 \mathrm{~min}$. Upon completion, the reaction contents were concentrated to afford $\mathrm{Me}_{2} \mathrm{AlCH}_{2} \mathrm{CH}_{2} \mathrm{AlMe}_{2}$ as a white free flowing powder $(0.206 \mathrm{~g}, 100 \%$ yield) that was stored under nitrogen prior to use. Note: the reagent was made freshly prior to its use in dithiane formation; stored reagent performed less adequately.

Ketone 13. Diels-Alder product 11 (0.512 g, 1.19 mmol, 1.0 equiv) was dissolved in 1,2dichloroethane $(10 \mathrm{~mL})$ and added to a flask containing solid $\mathrm{Me}_{2} \mathrm{AlCH}_{2} \mathrm{CH}_{2} \mathrm{AlMe}_{2}(0.736 \mathrm{~g}$, $3.57 \mathrm{mmol}, 3.0$ equiv) at $25{ }^{\circ} \mathrm{C}$. The resultant light yellow mixture was then warmed to $60{ }^{\circ} \mathrm{C}$ and stirred for $12 \mathrm{~h}$. Upon completion, the reaction contents were quenched by the slow and sequential addition of $\mathrm{Et}_{3} \mathrm{~N}(1 \mathrm{~mL})$ and Rochelle's salt $(1 \mathrm{~mL})$, poured into saturated aqueous $\mathrm{NaHCO}_{3}(25 \mathrm{~mL})$, and extracted with EtOAc $(3 \square 25 \mathrm{~mL})$. The combined organic layers were then washed with $\mathrm{H}_{2} \mathrm{O}(25 \mathrm{~mL})$ and brine $(25 \mathrm{~mL})$, dried $\left(\mathrm{Na}_{2} \mathrm{SO}_{4}\right)$, and concentrated to afford the desired dithiane intermediate as a yellow oil. This compound was immediately dissolved in THF $(10 \mathrm{~mL})$ and treated at $25^{\circ} \mathrm{C}$ with TBAF $(1.78 \mathrm{~mL}, 1.0 \mathrm{M}$ in THF, $1.78 \mathrm{mmol}, 1.5$ equiv). After 5 min of stirring at $25^{\circ} \mathrm{C}$, the reaction contents were poured into saturated aqueous $\mathrm{NH}_{4} \mathrm{Cl}$ 
$(25 \mathrm{~mL})$ and extracted with EtOAc $(3 \square 25 \mathrm{~mL})$. The combined organic layers were then washed with $\mathrm{H}_{2} \mathrm{O}(25 \mathrm{~mL})$ and brine $(25 \mathrm{~mL})$, dried $\left(\mathrm{Na}_{2} \mathrm{SO}_{4}\right)$, and concentrated. The resultant yellow residue was purified by flash column chromatography (silica gel, hexanes/EtOAc, 1:0 3:1) to give the desired intermediate $\left(0.304 \mathrm{~g}, 71 \%\right.$ yield) as a colorless oil. $\mathrm{R}_{f}=0.38$ (silica gel,

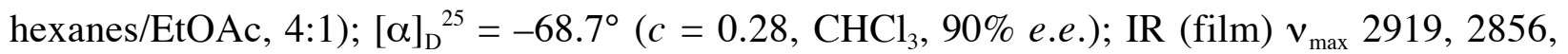
1713, 1437, 1279, 1175, 915, 859, $731 \mathrm{~cm}^{-1} ;{ }^{1} \mathrm{H}$ NMR (400 MHz, $\left.\mathrm{CDCl}_{3}\right) \square 5.23(\mathrm{~s}, 1 \mathrm{H}), 4.99$ $(\operatorname{app~t}, J=6.8 \mathrm{~Hz}, 1 \mathrm{H}), 4.87(\operatorname{app~t}, J=6.8 \mathrm{~Hz}, 1 \mathrm{H}), 3.23-3.18(\mathrm{~m}, 3 \mathrm{H}), 3.16-3.11(\mathrm{~m}, 1 \mathrm{H})$, $2.55(\mathrm{~m}, 2 \mathrm{H}), 2.37(\mathrm{~m}, 4 \mathrm{H}), 2.21-2.04(\mathrm{~m}, 6 \mathrm{H}), 1.88$ (ddd, $J=13.6,6.8,4.4 \mathrm{~Hz}, 1 \mathrm{H})$, 1.76-1.61 (m, $3 \mathrm{H}), 1.59(\mathrm{~s}, 3 \mathrm{H}), 1.50(\mathrm{~s}, 3 \mathrm{H}), 1.41(\mathrm{~m}, 1 \mathrm{H}) ;{ }^{13} \mathrm{C}$ NMR $\left(100 \mathrm{MHz}, \mathrm{CDCl}_{3}\right) \square$ 213.0, 136.2, 136.1, 125.6, 121.2, 59.7, 43.2, 42.9, 39.7, 39.1, 38.4, 38.3, 36.2, 35.1, 34.1, 30.6, 24.5, 19.3, 15.6; HRMS (MALDI-FTMS) calcd for $\mathrm{C}_{20} \mathrm{H}_{31} \mathrm{OS}_{2}^{+}\left[\mathrm{M}+\mathrm{H}^{+}\right] 351.1816$, found 351.1816. Next, a portion of the newly formed ketone $(0.132 \mathrm{~g}, 0.376 \mathrm{mmol}, 1.0$ equiv) was added dropwise as a solution in THF $(5 \mathrm{~mL})$ to a flask containing an excess amount of "hydrogen-free" Raney ${ }^{\square}$ nickel (prepared by stirring Raney ${ }^{\square} 2800$ nickel in acetone at reflux for 20 min and then removing the solvent with a Pasteur pipette), and the resultant slurry was stirred vigorously for $10 \mathrm{~h}$ at $25^{\circ} \mathrm{C}$. Upon completion, the reaction contents were filtered through Celite using several EtOAc washes to quantitate the transfer. The filtrate was then concentrated to afford ketone $\mathbf{1 3}\left(0.090 \mathrm{~g}, 92 \%\right.$ yield) as a white solid. 13: $\mathbf{R}_{f}=0.46$ (silica gel, hexanes/EtOAc, 4:1); m.p. $=82.0-83.5^{\circ} \mathrm{C} ;[\square]_{\mathrm{D}}{ }^{25}=-3.7^{\circ}\left(c=0.90, \mathrm{CHCl}_{3}, 90 \%\right.$ e.e. $)$; IR (film) $\square_{\max } 2923,2863,1713,1463,1382,1013,994,884,837,677 \mathrm{~cm}^{-1} ;{ }^{1} \mathrm{H}$ NMR $\left(400 \mathrm{MHz}, \mathrm{CDCl}_{3}\right) \square$ $4.81(\mathrm{~d}, J=11.2 \mathrm{~Hz}, 1 \mathrm{H}), 4.78(\mathrm{dd}, J=10.8,5.2 \mathrm{~Hz}, 1 \mathrm{H}), 2.46(\mathrm{td}, J=14.0,6.4 \mathrm{~Hz}, 1 \mathrm{H}), 2.36$ $(\mathrm{dq}, J=14.4,2.4 \mathrm{~Hz}, 1 \mathrm{H}), 2.26(\mathrm{~m}, 2 \mathrm{H}), 2.18(\mathrm{~m}, 2 \mathrm{H}), 2.10(\mathrm{~d}, J=14.0 \mathrm{~Hz}, 1 \mathrm{H}), 2.02(\mathrm{~m}, 1$ H), $1.96(\mathrm{dd}, J=11.2,4.8 \mathrm{~Hz}, 1 \mathrm{H}), 1.95(\mathrm{~m}, 2 \mathrm{H}), 1.82(\mathrm{~m}, 2 \mathrm{H}), 1.65(\mathrm{~m}, 1 \mathrm{H}), 1.58(\mathrm{~m}, 1 \mathrm{H})$, $1.54(\mathrm{~s}, 3 \mathrm{H}), 1.47(\mathrm{~s}, 3 \mathrm{H}), 1.19$ (q, $J=10.4 \mathrm{~Hz}, 1 \mathrm{H}), 1.02(\mathrm{~s}, 3 \mathrm{H}) ;{ }^{13} \mathrm{C} \mathrm{NMR}(100 \mathrm{MHz}$, $\left.\mathrm{CDCl}_{3}\right) \square 212.5,136.7,134.7,124.5,122.5,43.7,41.0,39.2,38.2,38.1,37.6,36.4,36.2,30.8$, 24.9, 19.8, 15.9, 12.3; HRMS (MALDI-FTMS) calcd for $\mathrm{C}_{20} \mathrm{H}_{31} \mathrm{ON}^{+}\left[\mathrm{M}+\mathrm{MeCN}^{+}\right]$302.2484, found 302.2478. 
Diazoketone 14. Ketone 13 (0.050 g, $0.192 \mathrm{mmol}, 1.0$ equiv) was dissolved in THF (2 $\mathrm{mL})$ and added dropwise over the course of $5 \mathrm{~min}$ to a solution of NaHMDS $(0.576 \mathrm{~mL}, 1.0 \mathrm{M}$ in THF, $0.576 \mathrm{mmol}, 3.0$ equiv) in THF $(2 \mathrm{~mL})$ at $-78^{\circ} \mathrm{C}$. After $30 \mathrm{~min}$ of stirring at $-78^{\circ} \mathrm{C}$, TMSCl (0.037 mL, $0.288 \mathrm{mmol}, 1.5$ equiv) was added in a single portion and the resultant mix was stirred for 30 additional min at $-78{ }^{\circ} \mathrm{C}$ and then was warmed to $0{ }^{\circ} \mathrm{C}$ over the course of 10 min. The reaction contents were quenched by the addition of saturated aqueous $\mathrm{NaHCO}_{3}(2$ $\mathrm{mL})$, poured into saturated aqueous $\mathrm{NaHCO}_{3}(25 \mathrm{~mL})$, and extracted with EtOAc $(3 \square 25 \mathrm{~mL})$. The combined organic layers were then washed with $\mathrm{H}_{2} \mathrm{O}(25 \mathrm{~mL})$ and brine $(25 \mathrm{~mL})$, dried $\left(\mathrm{Na}_{2} \mathrm{SO}_{4}\right)$, and concentrated. The resultant light yellow residue was taken up in $\mathrm{CH}_{2} \mathrm{Cl}_{2}(0.2 \mathrm{~mL})$ and treated with a solution of IBX $\bullet$ MPO (0.576 mmol, 3.0 equiv) in DMSO [prepared by stirring $\operatorname{IBX}^{6}(0.162 \mathrm{~g}, 0.576 \mathrm{mmol}, 3.0$ equiv) and 4-methoxypyridine $N$-oxide hydrate $(0.072 \mathrm{~g}, 0.576$ mmol, 3.0 equiv) in DMSO $(1 \mathrm{~mL})$ for $30 \mathrm{~min}$ at $25^{\circ} \mathrm{C}$ ]. The resultant yellow solution was stirred for $6 \mathrm{~h}$ at $25^{\circ} \mathrm{C}$. Upon completion, the reaction contents were poured into saturated aqueous $\mathrm{NaHCO}_{3}(25 \mathrm{~mL})$ and extracted with $\mathrm{Et}_{2} \mathrm{O}(3 \square 25 \mathrm{~mL})$, using exceedingly vigorous shaking to ensure complete material dissolution in the organic layer. The combined organic layers were then dried $\left(\mathrm{Na}_{2} \mathrm{SO}_{4}\right)$, and concentrated. The resultant light yellow residue was purified by flash column chromatography (silica gel, hexanes/EtOAc, 1:0 4:1) to give the desired $\square, \square$-unsaturated intermediate $\left(0.039 \mathrm{~g}, 79 \%\right.$ yield) as a colorless oil: $\mathrm{R}_{f}=0.54$ (silica gel, hexanes/EtOAc, 4:1); [ []$_{\mathrm{D}}{ }^{25}=+40.6^{\circ}\left(c=0.85, \mathrm{CHCl}_{3}, 90 \%\right.$ e.e. $)$; IR (film) $\square_{\max } 2923,2856$, $1681,1453,1387,1281,1223,1111,843,782 \mathrm{~cm}^{-1} ;{ }^{1} \mathrm{H}$ NMR $\left(400 \mathrm{MHz}, \mathrm{CDCl}_{3}\right) \square 6.66(\mathrm{~d}, J=$ $9.6 \mathrm{~Hz}, 1 \mathrm{H}), 5.94(\mathrm{~d}, J=9.6 \mathrm{~Hz}, 1 \mathrm{H}), 4.76(\mathrm{dd}, J=11.6,3.6 \mathrm{~Hz}, 1 \mathrm{H}), 4.60(\mathrm{~d}, J=9.6 \mathrm{~Hz}, 1 \mathrm{H})$, $2.46(\mathrm{~d}, J=12.8 \mathrm{~Hz}, 1 \mathrm{H}), 2.27(\mathrm{~m}, 3 \mathrm{H}), 2.19(\mathrm{~m}, 2 \mathrm{H}), 2.08-1.86(\mathrm{~m}, 5 \mathrm{H}), 1.71(\mathrm{t}, J=11.2 \mathrm{~Hz}$, $1 \mathrm{H}), 1.56(\mathrm{~s}, 3 \mathrm{H}), 1.46$ (s, $3 \mathrm{H}), 1.13(\mathrm{q}, J=10.4 \mathrm{~Hz}, 1 \mathrm{H}), 1.01(\mathrm{~s}, 3 \mathrm{H}) ;{ }^{13} \mathrm{C} \mathrm{NMR}(100 \mathrm{MHz}$, $\left.\mathrm{CDCl}_{3}\right) \square 200.5,161.9,136.1,135.5,128.6,125.0,122.8,40.5,39.8,38.9,38.1,37.6,36.4,28.4$, 25.4, 19.5, 17.4, 16.0; HRMS (MALDI-FTMS) calcd for $\mathrm{C}_{18} \mathrm{H}_{27} \mathrm{O}^{+}\left[\mathrm{M}+\mathrm{H}^{+}\right] 259.2062$, found 259.2069. After several repetitions of this oxidation procedure, a portion of the $\square, \square$-unsaturated ketone $(0.091 \mathrm{~g}, 0.352 \mathrm{mmol}, 1.0$ equiv) was taken up in benzene $(4 \mathrm{~mL})$ and treated sequentially 
at $25{ }^{\circ} \mathrm{C}$ with benzyltriethylammonium chloride $(0.049 \mathrm{~g}, 0.176 \mathrm{mmol}, 0.5$ equiv), 18 -crown-6 (5.0 mg, catalytic), 2,4,6-triisopropylbenzenesulfonyl azide ${ }^{7}$ (0.218 g, 0.704 mmol, 2.0 equiv), and $66 \%$ aqueous $\mathrm{KOH}(4 \mathrm{~mL})$. The resultant slurry was stirred vigorously at $40-45{ }^{\circ} \mathrm{C}$ for $48 \mathrm{~h}$, during which time additional 2,4,6-triisopropylbenzenesulfonyl azide (0.218 g, 0.704 mmol, 2.0 equiv) was added at $8 \mathrm{~h}$ intervals. Upon completion, the reaction contents were poured into $\mathrm{H}_{2} \mathrm{O}$ $(25 \mathrm{~mL})$ and extracted with $\mathrm{Et}_{2} \mathrm{O}(4 \square 25 \mathrm{~mL})$. The combined organic layers were then washed with $\mathrm{H}_{2} \mathrm{O}(2 \square 25 \mathrm{~mL})$ and brine $(25 \mathrm{~mL})$, dried $\left(\mathrm{Na}_{2} \mathrm{SO}_{4}\right)$, and concentrated. The resultant yellow residue was purified by flash column chromatography (silica gel, hexanes/EtOAc, 1:0 4:1) to give diazoketone $14(0.072 \mathrm{~g}, 72 \%$ yield $)$ as a yellow oil which was used immediately in subsequent chemistry along with a portion of recovered $\square, \square$-unsaturated ketone (0.008 g, 79\% yield based on recovered starting material).

○, $\square$-Unsaturated Ester 15. Diazoketone 14 (0.075 g, $0.264 \mathrm{mmol}, 1.0$ equiv) was dissolved in $\mathrm{MeOH}(12 \mathrm{~mL})$ and treated with $\mathrm{Et}_{3} \mathrm{~N}(0.1 \mathrm{~mL})$ at $25^{\circ} \mathrm{C}$. The resultant yellow solution was degassed with bubbling nitrogen for $45 \mathrm{~min}$, and then irradiated at $25^{\circ} \mathrm{C}$ for $3 \mathrm{~h}$ using a photoreactor equipped with a $450 \mathrm{~W}$ Hanovia UV lamp (medium pressure mercury lamp) and a Pyrex cooling jacket. Once this operation was complete, the reaction contents were concentrated, taken up in DBU $(2 \mathrm{~mL})$, and then heated at $115^{\circ} \mathrm{C}$ for $18 \mathrm{~h}$. Upon completion, the reaction contents were purified directly by flash column chromatography (silica gel, hexanes/EtOAc, 1:0 4:1) to give the ring-contracted $\square$, $\square$-unsaturated ester $15(0.052 \mathrm{~g}, 68 \%$ yield) as a colorless oil. 15: $\mathrm{R}_{f}=0.59$ (silica gel, hexanes/EtOAc, $\left.4: 1\right)$; $[\square]_{\mathrm{D}}{ }^{25}=-14.8^{\circ}(c=0.65$, $\mathrm{CHCl}_{3}, 90 \%$ e.e.); IR (film) $\square_{\max } 3020,2924,1702,1522,1507,1437,1086,930 \mathrm{~cm}^{-1} ;{ }^{1} \mathrm{H}$ NMR $\left(400 \mathrm{MHz}, \mathrm{CDCl}_{3}\right) \square 6.67(\mathrm{~m}, 1 \mathrm{H}), 5.19(\mathrm{dd}, J=11.6,4.4 \mathrm{~Hz}, 1 \mathrm{H}), 4.81(\mathrm{~d}, J=12.0 \mathrm{~Hz}, 1 \mathrm{H})$, $3.70(\mathrm{~s}, 3 \mathrm{H}), 2.54(\mathrm{~d}, J=11.2 \mathrm{~Hz}, 1 \mathrm{H}), 2.41(\mathrm{~m}, 2 \mathrm{H}), 2.27-2.03(\mathrm{~m}, 7 \mathrm{H}), 1.71(\mathrm{~s}, 3 \mathrm{H}), 1.66$ $(\mathrm{dd}, J=12.8,6.4 \mathrm{~Hz}, 1 \mathrm{H}), 1.55(\mathrm{dd}, J=14.8,10.0 \mathrm{~Hz}, 1 \mathrm{H}), 1.44$ (s, $3 \mathrm{H}), 1.38(\mathrm{~m}, 1 \mathrm{H}), 1.19$ $(\mathrm{s}, 3 \mathrm{H}) ;{ }^{13} \mathrm{C}$ NMR $\left(100 \mathrm{MHz}, \mathrm{CDCl}_{3}\right) \square 165.7,141.7,140.1,136.3,133.7,128.6,124.5,51.0$, 48.1, 47.1, 44.2, 40.4, 40.2, 38.0, 26.0, 24.3, 21.6, 15.4, 14.7; HRMS (ESI) calcd for $\mathrm{C}_{19} \mathrm{H}_{28} \mathrm{O}_{2}^{+}$ $\left[\mathrm{M}^{+}\right]$288.2090, found 288.2093. 
Palominol (3). Methyllithium (0.169 mL, 1.6 M in hexanes, $0.270 \mathrm{mmol}, 6.0$ equiv) was added dropwise over the course of $1 \mathrm{~min}$ to a solution of ester $15(0.013 \mathrm{~g}, 0.045 \mathrm{mmol}, 1.0$ equiv) in THF $(3 \mathrm{~mL})$ at $-20^{\circ} \mathrm{C}$. After $1 \mathrm{~h}$ of stirring at $-20^{\circ} \mathrm{C}$, the reaction contents were quenched by the addition of saturated aqueous $\mathrm{NaHCO}_{3}(2 \mathrm{~mL})$, poured into saturated aqueous $\mathrm{NaHCO}_{3}(25 \mathrm{~mL})$, and extracted with EtOAc $(3 \square 15 \mathrm{~mL})$. The combined organic layers were then washed with saturated aqueous $\mathrm{NaHCO}_{3}(15 \mathrm{~mL})$ and brine $(15 \mathrm{~mL})$, dried $\left(\mathrm{Na}_{2} \mathrm{SO}_{4}\right)$, and concentrated. The resultant light yellow residue was purified by flash column chromatography (silica gel, hexanes/EtOAc, 1:0 4:1) to give palominol (0.012 g, 92\% yield) as a colorless oil. 3: $\mathrm{R}_{f}=0.47$ (silica gel, hexanes/EtOAc, 4:1); $[\square]_{\mathrm{D}}{ }^{25}=-22.0^{\circ}\left(c=0.50, \mathrm{CHCl}_{3}\right.$, 90\% e.e. $)$; IR (film) $\square_{\max } 3260,2924,1507,1420,930 \mathrm{~cm}^{-1} ;{ }^{1} \mathrm{H}$ NMR (400 MHz, $\left.\mathrm{CDCl}_{3}\right) \square 5.47$ (m, $\left.1 \mathrm{H}\right), 5.21$ $(\mathrm{dd}, J=11.6,4.0 \mathrm{~Hz}, 1 \mathrm{H}), 4.85(\mathrm{~d}, J=10.4 \mathrm{~Hz}, 1 \mathrm{H}), 2.37(\mathrm{~d}, J=10.4 \mathrm{~Hz}, 1 \mathrm{H}), 2.34-2.20$ (m, $5 \mathrm{H}), 2.14-1.96(\mathrm{~m}, 4 \mathrm{H}), 1.93(\mathrm{dd}, J=16.4,3.2 \mathrm{~Hz}, 1 \mathrm{H}), 1.63$ (s, $3 \mathrm{H}), 1.56$ (dd, $J=12.4,5.2$ $\mathrm{Hz}, 1 \mathrm{H}), 1.50(\mathrm{~s}, 3 \mathrm{H}), 1.42(\mathrm{~s}, 3 \mathrm{H}), 1.38(\mathrm{~s}, 3 \mathrm{H}), 1.24(\mathrm{~m}, 1 \mathrm{H}), 1.17(\mathrm{~s}, 3 \mathrm{H}) ;{ }^{13} \mathrm{C}$ NMR $(100$ $\left.\mathrm{MHz}, \mathrm{CDCl}_{3}\right) \square 154.0,134.6,133.4,128.6,125.4,122.6,71.6,47.8,47.4,46.1,40.7,40.0,38.1$, 31.8, 26.1, 24.4, 22.7, 16.2, 15.4; HRMS (ESI) calcd for $\mathrm{C}_{20} \mathrm{H}_{32} \mathrm{O}^{+}\left[\mathrm{M}^{+}\right] 288.2453$, found $288.2454 .^{8}$

Dolabellatrienone (2). Allyl alcohol $3(5.1 \mathrm{mg}, 0.017 \mathrm{mmol}, 1.0$ equiv) was taken up in $\mathrm{CH}_{2} \mathrm{Cl}_{2}(1 \mathrm{~mL})$ and treated sequentially at $25^{\circ} \mathrm{C}$ with powdered $4 \AA$ molecular sieves $(15 \mathrm{mg})$ and pyridinium dichromate $(13.3 \mathrm{mg}, 0.035 \mathrm{mmol}, 2.0$ equiv). The resultant orange-brown slurry was stirred at $25^{\circ} \mathrm{C}$ for $5 \mathrm{~h}$. Upon completion, the reaction contents were diluted with $\mathrm{CH}_{2} \mathrm{Cl}_{2}(2 \mathrm{~mL})$, filtered through a plug of silica gel (using several $\mathrm{CH}_{2} \mathrm{Cl}_{2}$ washes), and concentrated. The resultant colorless residue was purified by preparative TLC (silica gel, hexanes/EtOAc, 4:1) to give dolabellatrienone $\left(3.4 \mathrm{mg}, 67 \%\right.$ yield) as a colorless oil. $\mathbf{2}: \mathrm{R}_{f}=$ 0.49 (silica gel, hexanes/EtOAc, $4: 1) ;[\square]_{\mathrm{D}}{ }^{25}=+28.0^{\circ}\left(c=0.05, \mathrm{CHCl}_{3}, 90 \%\right.$ e.e. $)$; IR (film) $\square_{\max }$ 2972, 2851, 1703, 1622, 1440, 1405, 1384, 1274, 1181, $1171 \mathrm{~cm}^{-1} ;{ }^{1} \mathrm{H}$ NMR $\left(400 \mathrm{MHz}, \mathrm{CDCl}_{3}\right)$ $\square 5.24(\mathrm{dd}, J=12.0,6.0 \mathrm{~Hz}, 1 \mathrm{H}), 4.92(\mathrm{~d}, J=9.6 \mathrm{~Hz}, 1 \mathrm{H}), 2.82(\mathrm{~d}, J=12.8 \mathrm{~Hz}, 1 \mathrm{H}), 2.38(\mathrm{~d}, J$ $=18.0 \mathrm{~Hz}, 1 \mathrm{H}), 2.37-2.22(\mathrm{~m}, 3 \mathrm{H}), 2.22(\mathrm{~s}, 3 \mathrm{H}), 2.18-2.06(\mathrm{~m}, 5 \mathrm{H}), 1.83(\mathrm{~s}, 3 \mathrm{H}), 1.64(\mathrm{~s}, 3$ 
$\mathrm{H}), 1.64-1.55(\mathrm{~m}, 2 \mathrm{H}), 1.47(\mathrm{~m}, 1 \mathrm{H}), 1.45(\mathrm{~s}, 3 \mathrm{H}), 1.23(\mathrm{~s}, 3 \mathrm{H}) ;{ }^{13} \mathrm{C} \mathrm{NMR}\left(100 \mathrm{MHz}, \mathrm{CDCl}_{3}\right)$

$\square 207.4,148.3,138.0,135.7,131.8,130.4,124.8,54.9,41.5,41.1,40.1,39.8,38.2,28.0,24.5$, 24.3, 23.2, 21.4, 16.2, 15.5; HRMS (ESI) calcd for $\mathrm{C}_{20} \mathrm{H}_{30} \mathrm{O}^{+}\left[\mathrm{M}^{+}\right]$286.2297, found 286.2301. ${ }^{9}$

Ketone 16. To a solution of ester $15(8.0 \mathrm{mg}, 0.028 \mathrm{mmol}, 1.0$ equiv) in THF (1 mL) at $-30{ }^{\circ} \mathrm{C}$ was added L-Selectride ${ }^{\square}(0.111 \mathrm{~mL}, 1.0 \mathrm{M}$ in THF, $0.111 \mathrm{mmol}, 4.0$ equiv). The resultant solution was then warmed to $0{ }^{\circ} \mathrm{C}$ and stirred for $1.5 \mathrm{~h}$. Upon completion, the reaction contents were quenched by the slow addition of saturated aqueous Rochelle's salt $(1 \mathrm{~mL})$ and poured into EtOAc $(15 \mathrm{~mL})$. The organic layer was washed with $\mathrm{H}_{2} \mathrm{O}(2 \square 15 \mathrm{~mL})$, dried $\left(\mathrm{Na}_{2} \mathrm{SO}_{4}\right)$, and concentrated. The resultant light yellow oil was purified by flash column chromatography (silica gel, hexanes/EtOAc, 1:0 4:1) to give the desired intermediate (7.0 mg, $87 \%$ yield) as a colorless oil. Next, the newly formed ester (7.0 mg, $0.024 \mathrm{mmol}, 1.0$ equiv) was taken up in THF $(1 \mathrm{~mL})$ and treated at $0{ }^{\circ} \mathrm{C}$ with LDA $(0.121 \mathrm{~mL}, 1.0 \mathrm{M}$ in THF, $0.121 \mathrm{mmol}$, 1.0 equiv). After $30 \mathrm{~min}$ of stirring at $0{ }^{\circ} \mathrm{C}$, a balloon filled with oxygen gas and equipped with a long syringe was inserted into the reaction vessel, and oxygen gas was bubbled directly into the solution for $10 \mathrm{~min}$ at $0{ }^{\circ} \mathrm{C}$. Triethylphosphite ( 2 drops) was then added, and after 5 min of additional stirring at $0{ }^{\circ} \mathrm{C}$, the reaction contents were poured into saturated aqueous $\mathrm{NH}_{4} \mathrm{Cl}(10$ $\mathrm{mL})$ and extracted with EtOAc $(3 \square 15 \mathrm{~mL})$. The combined organic layers were then dried $\left(\mathrm{Na}_{2} \mathrm{SO}_{4}\right)$ and concentrated. The resultant light yellow oil was purified by flash column chromatography (silica gel, hexanes/EtOAc, 1:0 4:1) to give the desired tertiary alcohol intermediate $(6.0 \mathrm{mg}, 81 \%$ yield $)$ as a colorless oil. Finally, $\mathrm{LiAlH}_{4}(0.059 \mathrm{~mL}, 1.0 \mathrm{M}$ in THF, $0.059 \mathrm{mmol}, 3.0$ equiv) was added to a solution of the newly-formed tertiary alcohol (6.0 $\mathrm{mg}$, $0.020 \mathrm{mmol}, 1.0$ equiv) in THF $(1 \mathrm{~mL})$ at $25^{\circ} \mathrm{C}$. The resultant solution was stirred at $25^{\circ} \mathrm{C}$ for $10 \mathrm{~min}$, quenched by the slow addition of saturated aqueous Rochelle's salt ( $2 \mathrm{~mL})$, and poured into EtOAc $(15 \mathrm{~mL})$. The organic layer was washed with $\mathrm{H}_{2} \mathrm{O}(2 \square 15 \mathrm{~mL})$, dried $\left(\mathrm{MgSO}_{4}\right)$, and concentrated. The resultant colorless residue was taken up in $\mathrm{CH}_{2} \mathrm{Cl}_{2}(1 \mathrm{~mL})$ and treated at $25^{\circ} \mathrm{C}$ with excess $\mathrm{NaIO}_{4}$ on silica gel. ${ }^{10}$ After 15 min of stirring at $25{ }^{\circ} \mathrm{C}$, the reaction contents were filtered, concentrated, and purified directly by flash column chromatography (silica gel, 
hexanes/EtOAc, 1:0 3:1) to give ketone 16 (3.5 mg, 73\% yield, 51\% overall yield from 15) as a colorless oil. 16: $\mathrm{R}_{f}=0.46$ (silica gel, hexanes/EtOAc, $\left.3: 1\right) ;[\square]_{\mathrm{D}}{ }^{25}=+40.0^{\circ}\left(c=0.05, \mathrm{CHCl}_{3}\right.$, $90 \%$ e.e.); all other physical and spectral data were in complete agreement with that published by these laboratories. ${ }^{11}$

\section{Preparation of the Substrates in Table 1.}

Aldehyde 21. To a solution of methyl $O$-methylpodocarpate $(0.334 \mathrm{~g}, 1.11 \mathrm{mmol}, 1.0$ equiv) in THF (5 mL) at $25^{\circ} \mathrm{C}$ was added solid $\mathrm{LiAlH}_{4}(0.168 \mathrm{~g}, 4.42 \mathrm{mmol}, 4.0$ equiv). The resultant slurry was stirred at $25^{\circ} \mathrm{C}$ for $12 \mathrm{~h}$, and then heated at $67^{\circ} \mathrm{C}$ for $2 \mathrm{~h}$. Upon completion, the reaction contents were cooled to $0{ }^{\circ} \mathrm{C}$, quenched by the slow addition of saturated aqueous Rochelle's salt $(5 \mathrm{~mL})$, and poured into EtOAc $(20 \mathrm{~mL})$. The organic layer was washed with $\mathrm{H}_{2} \mathrm{O}(2 \square 25 \mathrm{~mL})$, dried $\left(\mathrm{MgSO}_{4}\right)$, and concentrated to give the desired alcohol $(0.297 \mathrm{~g}, 98 \%$ yield) as a white solid. Next, oxalyl chloride $(0.370 \mathrm{~mL}, 4.24 \mathrm{mmol}, 4.0$ equiv) was added to a solution of DMSO (0.677 mL, $8.66 \mathrm{mmol}, 8.0$ equiv) in $\mathrm{CH}_{2} \mathrm{Cl}_{2}(4 \mathrm{~mL})$ at $-78{ }^{\circ} \mathrm{C}$. After $10 \mathrm{~min}$ of stirring at $-78{ }^{\circ} \mathrm{C}$, the newly-formed intermediate alcohol $(0.297 \mathrm{~g}, 1.06 \mathrm{mmol}, 1.0$ equiv) was added dropwise as a solution in $\mathrm{CH}_{2} \mathrm{Cl}_{2}(4 \mathrm{~mL})$ at $-78{ }^{\circ} \mathrm{C}$. Following $30 \mathrm{~min}$ of stirring at -78 ${ }^{\circ} \mathrm{C}, \mathrm{Et}_{3} \mathrm{~N}$ (2.70 mL, $19.6 \mathrm{mmol}, 20$ equiv) was added and the mixture was allowed to warm slowly to $25{ }^{\circ} \mathrm{C}$. The reaction contents were then poured into $\mathrm{H}_{2} \mathrm{O}(50 \mathrm{~mL})$ and extracted with $\mathrm{CH}_{2} \mathrm{Cl}_{2}(3 \square 25 \mathrm{~mL})$. The combined organic layers were then dried $\left(\mathrm{MgSO}_{4}\right)$ and concentrated. The resultant yellow residue was purified by flash column chromatography (silica gel, hexanes/EtOAc, 1:0 4:1) to give aldehyde 21 (0.271 g, 92\% yield) as a fluffy white solid. 21: $\mathrm{R}_{f}=0.54$ (silica gel, hexanes/EtOAc, 4:1); IR (film) $\square_{\max } 2927,2831,1711,1611,1574,1501$, 1262, 1173, 1050, 899, $864 \mathrm{~cm}^{-1}$; ${ }^{1} \mathrm{H}$ NMR (400 MHz, $\left.\mathrm{CDCl}_{3}\right) \square 9.83(\mathrm{~s}, 1 \mathrm{H}), 6.98(\mathrm{~d}, J=8.4$ $\mathrm{Hz}, 1 \mathrm{H}), 6.80(\mathrm{~d}, J=2.8 \mathrm{~Hz}, 1 \mathrm{H}), 6.68(\mathrm{dd}, J=8.4,2.8 \mathrm{~Hz}, 1 \mathrm{H}), 3.77(\mathrm{~s}, 3 \mathrm{H}), 2.93(\mathrm{dd}, J=$ 16.8, $6.0 \mathrm{~Hz}, 1 \mathrm{H}), 2.81(\mathrm{ddd}, J=18.4,12.0,6.8 \mathrm{~Hz}, 1 \mathrm{H}), 2.23(\mathrm{~m}, 3 \mathrm{H}), 2.01$ (m, $1 \mathrm{H}), 1.77$ (dt, $J=12.8,4.0 \mathrm{~Hz}, 1 \mathrm{H}), 1.70(\mathrm{~m}, 3 \mathrm{H}), 1.41(\mathrm{dd}, J=13.2,4.0 \mathrm{~Hz}, 1 \mathrm{H}), 1.10(\mathrm{~s}, 3 \mathrm{H}), 1.07$ (s, 3 $\mathrm{H}) ;{ }^{13} \mathrm{C}$ NMR $\left(100 \mathrm{MHz}, \mathrm{CDCl}_{3}\right) \square 205.7,157.8,148.8,129.9,126.8,111.3,110.7,55.2,51.9$, 
48.6, 38.4, 38.2, 33.8, 30.4, 24.2, 24.0, 19.2, 18.9; HRMS (ESI) calcd for $\mathrm{C}_{18} \mathrm{H}_{24} \mathrm{O}_{2}^{+}\left[\mathrm{M}^{+}\right]$ 272.1776 , found $272.1777 .^{12}$

Aldehyde 23. To a solution of $i$ - $\mathrm{Pr}_{2} \mathrm{NH}(2.19 \mathrm{~mL}, 15.6 \mathrm{mmol}, 1.2$ equiv) in THF (15 mL) at $0{ }^{\circ} \mathrm{C}$ was slowly added $n$-BuLi $(8.94 \mathrm{~mL}, 1.6 \mathrm{M}$ in hexanes, $14.3 \mathrm{mmol}, 1.1$ equiv). After 10 min of stirring at $0{ }^{\circ} \mathrm{C}$, the reaction contents were cooled to $-78{ }^{\circ} \mathrm{C}$ and treated with a solution of methyl vinyl ketone (1.08 mL, $13.0 \mathrm{mmol}, 1.0$ equiv) in THF (5 mL). After $30 \mathrm{~min}$ of stirring at $-78{ }^{\circ} \mathrm{C}$, TIPSOTf $(3.50 \mathrm{~mL}, 13.0 \mathrm{mmol}, 1.0$ equiv) was added, and the resultant colorless solution was warmed slowly to $0{ }^{\circ} \mathrm{C}$ over the course of $1 \mathrm{~h}$. Upon completion, the reaction contents were quenched by the addition of saturated aqueous $\mathrm{NaHCO}_{3}(10 \mathrm{~mL})$, poured into saturated aqueous $\mathrm{NaHCO}_{3}(25 \mathrm{~mL})$, and extracted with hexanes $(3 \square 25 \mathrm{~mL})$. The combined organic layers were then washed with brine $(25 \mathrm{~mL})$, dried $\left(\mathrm{Na}_{2} \mathrm{SO}_{4}\right)$, and concentrated. The resultant light yellow oil was purified by flash column chromatography $\left(\mathrm{Et}_{3} \mathrm{~N}\right.$-deactivated silica gel, hexanes/EtOAc, 1:0 9:1) to give TIPS-diene (1.81 g, 62\% yield) as a colorless oil. Next, to a solution of freshly prepared catalyst $(S)-4(0.237 \mathrm{mmol}, 0.1$ equiv) in toluene $(2 \mathrm{~mL})$ at -78 ${ }^{\circ} \mathrm{C}$ was added sequentially a solution of TIPS-diene $(0.541 \mathrm{~g}, 2.39 \mathrm{mmol}, 1.0$ equiv) in toluene (1 $\mathrm{mL})$ and a solution of 1-cyclohexene-1-carboxaldehyde (0.263 g, $2.39 \mathrm{mmol}, 1.0$ equiv) in toluene $(1 \mathrm{~mL})$. The resultant light yellow reaction mixture was stirred for $30 \mathrm{~min}$ at $-78{ }^{\circ} \mathrm{C}$, allowed to warm slowly to $5^{\circ} \mathrm{C}$, and stirred for an additional $14 \mathrm{~h}$ at $5{ }^{\circ} \mathrm{C}$. Upon completion, the reaction contents were quenched by the addition of $\mathrm{Et}_{3} \mathrm{~N}(0.5 \mathrm{~mL})$, warmed to $25{ }^{\circ} \mathrm{C}$, concentrated, and purified directly by flash column chromatography $\left(\mathrm{Et}_{3} \mathrm{~N}\right.$-deactivated silica gel, hexanes/EtOAc, 1:0 9:1) to give Diels-Alder product 23 (0.479 g, 60\% yield) as a colorless oil. 23: $\mathrm{R}_{f}=0.50$ (silica gel, hexanes/EtOAc, 9:1); IR (film) $\square_{\max } 2929,2865,1727,1675,1465$, 1376, 1245, 1204, 884, $685 \mathrm{~cm}^{-1} ;{ }^{1} \mathrm{H}$ NMR (400 MHz, $\mathrm{CDCl}_{3}$ ) $\square 9.42$ (s, $\left.1 \mathrm{H}\right), 4.76$ (m, $1 \mathrm{H}$ ), 2.37-2.28 (m, 2 H), $2.11(\mathrm{~m}, 1 \mathrm{H}), 2.03(\mathrm{~m}, 1 \mathrm{H}), 1.77-1.63(\mathrm{~m}, 2 \mathrm{H}), 1.60(\mathrm{dt}, J=12.8,3.6 \mathrm{~Hz}$, $1 \mathrm{H}), 1.42-1.28(\mathrm{~m}, 6 \mathrm{H}), 1.17-1.04(\mathrm{~m}, 21 \mathrm{H}) ;{ }^{13} \mathrm{C}$ NMR (100 MHz, $\left.\mathrm{CDCl}_{3}\right) \square$ 206.9, 149.5, 99.4, 48.6, 34.6, 34.1, 30.2, 28.8, 25.4, 25.1, 21.5, 18.3, 12.9; HRMS (MALDI-FTMS) calcd for $\mathrm{C}_{20} \mathrm{H}_{37} \mathrm{O}_{2} \mathrm{Si}^{+}\left[\mathrm{M}+\mathrm{H}^{+}\right]$337.2563, found 337.2549. ${ }^{13}$ 
Aldehyde 25. Aldehyde $\mathbf{2 5}$ was synthesized in a manner analogous to the preparation of 12 from 10, substituting TBSOTf for TIPSOTf. Overall, this sequence afforded 25 as a colorless oil in $58 \%$ overall yield from the intermediate methyl ketone. 25: $\mathrm{R}_{f}=0.38$ (silica gel, hexanes/EtOAc, 19:1); $[\square]_{\mathrm{D}}{ }^{25}=-55.4^{\circ}\left(c=1.0, \mathrm{CHCl}_{3}, 85 \%\right.$ e.e. $)$; IR (film) $\square_{\max } 2929,2858$, $1715,1669,1449,1374,1264,1202,1181,909,837,731 \mathrm{~cm}^{-1} ;{ }^{1} \mathrm{H}$ NMR $\left(400 \mathrm{MHz}, \mathrm{CDCl}_{3}\right)$ $9.76(\mathrm{~s}, 1 \mathrm{H}), 4.94(\operatorname{app~t}, J=6.0 \mathrm{~Hz}, 1 \mathrm{H}), 4.82(\operatorname{app~t}, J=6.4 \mathrm{~Hz}, 1 \mathrm{H}), 4.81(\mathrm{~s}, 1 \mathrm{H}), 2.47$ (dt, $J$ $=8.4,2.8 \mathrm{~Hz}, 1 \mathrm{H}), 2.37(\mathrm{dd}, J=14.4,8.4 \mathrm{~Hz}, 1 \mathrm{H}), 2.22-1.98(\mathrm{~m}, 7 \mathrm{H}), 1.93(\mathrm{dd}, J=13.2,4.8$ Hz, 1 H), 1.87-1.79 (m, 3 H), 1.56 (s, 3 H), 1.43 (s, 3 H), 1.42-1.37 (m, 2 H), 0.91 (s, 9 H), 0.13 (s, $3 \mathrm{H}), 0.12$ (s, $3 \mathrm{H}) ;{ }^{13} \mathrm{C}$ NMR (100 MHz, $\left.\mathrm{CDCl}_{3}\right) \square 206.9$, 149.3, 136.5, 135.4, 126.0, 121.9, $108.4,51.4,39.4,37.1,35.2,31.1,30.0,29.8,26.8,25.6,25.0,18.0,17.2,15.7,-4.3,-4.5$; HRMS (ESI) calcd for $\mathrm{C}_{24} \mathrm{H}_{40} \mathrm{O}_{2} \mathrm{Si}^{+}\left[\mathrm{M}^{+}\right]$388.2798, found 388.2815.

General procedure for the synthesis of dithianes from ketones and aldehydes using $\mathbf{M e}_{2} \mathrm{AlSCH}_{2} \mathrm{CH}_{2} \mathrm{AlMe}_{2}$. The substrate $(0.250 \mathrm{mmol}, 1.0$ equiv) was dissolved in 1,2dichloroethane $(5 \mathrm{~mL})$ and added to a flask containing solid $\mathrm{Me}_{2} \mathrm{AlCH}_{2} \mathrm{CH}_{2} \mathrm{AlMe}_{2}(0.154 \mathrm{~g}$, $0.750 \mathrm{mmol}, 3.0$ equiv) at $25^{\circ} \mathrm{C}$. The resultant light yellow mixture was then warmed to $60{ }^{\circ} \mathrm{C}$ and stirred for 2-12 h. Upon completion, the reaction contents were quenched by the slow and sequential addition of $\mathrm{Et}_{3} \mathrm{~N}(1 \mathrm{~mL})$ and Rochelle's salt $(1 \mathrm{~mL})$, poured into saturated aqueous $\mathrm{NaHCO}_{3}(25 \mathrm{~mL})$, and extracted with EtOAc $(3 \square 25 \mathrm{~mL})$. The combined organic layers were then washed with $\mathrm{H}_{2} \mathrm{O}(25 \mathrm{~mL})$ and brine $(25 \mathrm{~mL})$, dried $\left(\mathrm{Na}_{2} \mathrm{SO}_{4}\right)$, and concentrated. The resultant residue was purified by flash column chromatography (silica gel, hexanes/EtOAc, 1:0 4:1) to afford the desired dithiane products in the yield indicated in Table 1.

18: $R_{f}=0.49$ (silica gel, hexanes/EtOAc, 9:1); IR (film) $\square_{\max } 2937,2865,1445,1426$, 1364, 1273, 1237, 1185, 1027, $837 \mathrm{~cm}^{-1} ;{ }^{1} \mathrm{H}$ NMR (400 MHz, $\mathrm{CDCl}_{3}$ ) $\square 3.28$ (m, $\left.4 \mathrm{H}\right), 2.16$ (m, 2 H), $1.87(\mathrm{td}, J=13.2,3.6 \mathrm{~Hz}, 2 \mathrm{H}), 1.78(\mathrm{~m}, 2 \mathrm{H}), 1.29(\mathrm{dq}, J=13.6,3.6 \mathrm{~Hz}, 2 \mathrm{H}), 1.00(\mathrm{dt}, J=$ 12.0, $2.8 \mathrm{~Hz}, 1 \mathrm{H}), 0.85$ (s, $9 \mathrm{H}) ;{ }^{13} \mathrm{C} \mathrm{NMR}\left(100 \mathrm{MHz}, \mathrm{CDCl}_{3}\right) \square$ 68.7, 46.8, 43.1, 38.7, 38.0, 32.3, 27.5, 27.1; HRMS (ESI) calcd for $\mathrm{C}_{12} \mathrm{H}_{22} \mathrm{~S}_{2}^{+}\left[\mathrm{M}^{+}\right] 230.1163$, found 230.1169. 
20: $\mathrm{R}_{f}=0.43$ (silica gel, hexanes/EtOAc, 9:1); IR (film) $\square_{\max } 2917,2856,1609,1461$, 1376, 1275, 1167, 1032, 855, 812, 782, 704, $687 \mathrm{~cm}^{-1} ;{ }^{1} \mathrm{H}$ NMR (400 MHz, $\left.\mathrm{CDCl}_{3}\right) \square 6.84(\mathrm{~s}, 2$ $\mathrm{H}), 6.32(\mathrm{~s}, 1 \mathrm{H}), 3.52(\mathrm{~m}, 2 \mathrm{H}), 3.36(\mathrm{~m}, 2 \mathrm{H}), 2.56(\mathrm{~s}, 6 \mathrm{H}), 2.26(\mathrm{~s}, 3 \mathrm{H}) ;{ }^{13} \mathrm{C} \mathrm{NMR}(100 \mathrm{MHz}$, $\left.\mathrm{CDCl}_{3}\right) \square 138.3,137.5,130.3,128.6,51.5,40.2,20.9,20.7$; HRMS (ESI) calcd for $\mathrm{C}_{12} \mathrm{H}_{16} \mathrm{~S}_{2}^{+}$ $\left[\mathrm{M}^{+}\right]$224.0693, found 224.0686.

22: $\mathrm{R}_{f}=0.58$ (silica gel, hexanes/EtOAc, 4:1); IR (film) $\square_{\max } 2923,2833,1609,1574$, 1503, 1465, 1376, 1275, 1254, 1173, 1044, 909, 851, $733 \mathrm{~cm}^{-1} ;{ }^{1} \mathrm{H}$ NMR (400 MHz, $\left.\mathrm{CDCl}_{3}\right)$ $6.94(\mathrm{~d}, J=8.0 \mathrm{~Hz}, 1 \mathrm{H}), 6.81(\mathrm{~d}, J=2.4 \mathrm{~Hz}, 1 \mathrm{H}), 6.66(\mathrm{dd}, J=8.4,2.4 \mathrm{~Hz}, 1 \mathrm{H}), 5.30(\mathrm{~s}, 1 \mathrm{H})$, 3.77 (s, $3 \mathrm{H}), 3.27-3.00$ (m, $4 \mathrm{H}), 2.87$ (dd, $J=16.8,4.8 \mathrm{~Hz}, 1 \mathrm{H}), 2.70$ (ddd, $J=18.8,12.4,6.4$ $\mathrm{Hz}, 1 \mathrm{H}), 2.31(\mathrm{dt}, J=12.0,2.4 \mathrm{~Hz}, 1 \mathrm{H}), 2.08(\mathrm{dd}, J=13.2,6.4 \mathrm{~Hz}, 1 \mathrm{H}), 2.00(\mathrm{dt}, J=12.4,2.4$ $\mathrm{Hz}, 1 \mathrm{H}), 1.86-1.76(\mathrm{~m}, 2 \mathrm{H}), 1.65$ (dt, $J=10.4,3.6 \mathrm{~Hz}, 1 \mathrm{H}), 1.59$ (dd, $J=12.8,1.6 \mathrm{~Hz}, 1 \mathrm{H})$, $1.42(\mathrm{~m}, 2 \mathrm{H}), 1.39$ (s, $3 \mathrm{H}), 1.21$ (s, $3 \mathrm{H}) ;{ }^{13} \mathrm{C}$ NMR (100 MHz, $\left.\mathrm{CDCl}_{3}\right) \square$ 157.8, 151.1, 129.7, $127.3,111.1,110.8,60.1,55.2,53.7,42.7,41.2,39.9,39.8,38.5,36.7,31.3,25.4,23.9,21.3$, 18.8; HRMS (ESI) calcd for $\mathrm{C}_{20} \mathrm{H}_{28} \mathrm{OS}_{2}^{+}\left[\mathrm{M}^{+}\right]$348.1582, found 348.1583.

24: $\mathrm{R}_{f}=0.61$ (silica gel, hexanes/EtOAc, 9:1); IR (film) $\square_{\max } 2925,2865,1673,1465$, 1376, 1241, 1196, 1071, 996, 882, 847, 797, $683 \mathrm{~cm}^{-1} ;{ }^{1} \mathrm{H}$ NMR (400 MHz, $\left.\mathrm{CDCl}_{3}\right) \square 5.19(\mathrm{~s}, 1$ H), 4.66 (m, $1 \mathrm{H}), 3.22-3.12(\mathrm{~m}, 2 \mathrm{H}), 3.08(\mathrm{~m}, 2 \mathrm{H}), 2.47$ (m, $2 \mathrm{H}), 1.83$ (dd, $J=18.0,3.6 \mathrm{~Hz}, 1$ $\mathrm{H}), 1.76(\mathrm{~m}, 1 \mathrm{H}), 1.64(\mathrm{~m}, 3 \mathrm{H}), 1.57-1.35(\mathrm{~m}, 6 \mathrm{H}), 1.13(\mathrm{~m}, 21 \mathrm{H}) ;{ }^{13} \mathrm{C} \mathrm{NMR}(100 \mathrm{MHz}$, $\left.\mathrm{CDCl}_{3}\right) \square 148.6,99.0,61.7,39.9,39.0,38.8,38.1,33.9,29.9,28.7,27.8,25.7,21.6,18.0,12.6$; HRMS (MALDI-FTMS) calcd for $\mathrm{C}_{22} \mathrm{H}_{41} \mathrm{OS}_{2} \mathrm{Si}^{+}\left[\mathrm{M}+\mathrm{H}^{+}\right]$413.2368, found 413.2355.

26: $\mathrm{R}_{f}=0.45$ (silica gel, hexanes/EtOAc, 19:1); IR (film) $\square_{\max } 2925,2856,1669,1449$, 1252, 1198, 1177, 837, $778 \mathrm{~cm}^{-1}$; ${ }^{1} \mathrm{H}$ NMR (400 MHz, $\left.\mathrm{CDCl}_{3}\right) \square 5.02$ (s, $\left.1 \mathrm{H}\right), 4.95$ (br d, J = 8.4 Hz, $1 \mathrm{H}), 4.79$ (dd, $J=10.0,4.8 \mathrm{~Hz}, 1 \mathrm{H}), 4.62$ (br s, $1 \mathrm{H}), 3.12$ (m, $3 \mathrm{H}), 3.01$ (m, $1 \mathrm{H}), 2.69$ $(\mathrm{dd}, J=14.4,10.0 \mathrm{~Hz}, 1 \mathrm{H}), 2.44(\mathrm{br} \mathrm{d}, J=8.8 \mathrm{~Hz}, 1 \mathrm{H}), 2.38-2.19$ (m, $3 \mathrm{H}), 2.12-1.96$ (m, 6 H), $1.85(\mathrm{~m}, 1 \mathrm{H}), 1.71(\mathrm{dd}, J=12.8,9.6 \mathrm{~Hz}, 1 \mathrm{H}), 1.59(\mathrm{~s}, 3 \mathrm{H}), 1.49(\mathrm{~s}, 3 \mathrm{H}), 1.41(\mathrm{~m}, 1 \mathrm{H})$, $1.14(\mathrm{~m}, 1 \mathrm{H}), 0.92(\mathrm{~s}, 9 \mathrm{H}), 0.15(\mathrm{~s}, 6 \mathrm{H})$. For the purposes of complete characterization, compound 26 was converted to its corresponding ketone intermediate using 1.0 M TBAF in THF 
as described earlier for the conversion of 12 to 13; physical and spectral data for both samples were in complete agreement. Note: reaction of 25 with $\mathrm{Me}_{2} \mathrm{AlCH}_{2} \mathrm{CH}_{2} \mathrm{AlMe}_{2}$ for prolonged periods (i.e. more than $2 \mathrm{~h}$ ) led to partial deprotection of the silyl enol ether and formation of the corresponding bis-dithiane.

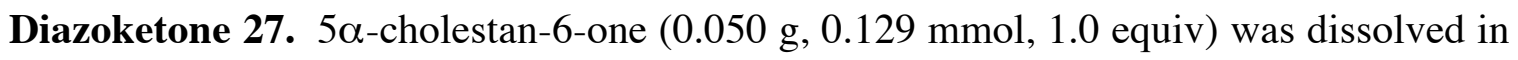
THF (2 mL) and added dropwise over the course of $5 \mathrm{~min}$ to a solution of NaHMDS $(0.388 \mathrm{~mL}$, $1.0 \mathrm{M}$ in THF, $0.388 \mathrm{mmol}, 3.0$ equiv) in THF $(3 \mathrm{~mL})$ at $-78^{\circ} \mathrm{C}$. After $30 \mathrm{~min}$ of stirring at -78 ${ }^{\circ} \mathrm{C}$, TMSCl (0.025 mL, $0.194 \mathrm{mmol}, 1.5$ equiv) was added in a single portion and the resultant mix was stirred for 30 additional min at $-78{ }^{\circ} \mathrm{C}$ and then was warmed to $0{ }^{\circ} \mathrm{C}$ over the course of $10 \mathrm{~min}$. The reaction contents were quenched by the addition of saturated aqueous $\mathrm{NaHCO}_{3}(10$ $\mathrm{mL})$, poured into saturated aqueous $\mathrm{NaHCO}_{3}(25 \mathrm{~mL})$, and extracted with hexanes $(3 \square 25 \mathrm{~mL})$. The combined organic layers were then washed with $\mathrm{H}_{2} \mathrm{O}(25 \mathrm{~mL})$ and brine $(25 \mathrm{~mL})$, dried $\left(\mathrm{Na}_{2} \mathrm{SO}_{4}\right)$, and concentrated. The resultant light yellow solid was then dissolved in THF (2 mL), cooled to $-78{ }^{\circ} \mathrm{C}$, and treated with a solution of $\mathrm{PhSeCl}(0.030 \mathrm{~g}, 0.155 \mathrm{mmol}, 1.2$ equiv) in THF $(2 \mathrm{~mL})$ added dropwise over the course of $2 \mathrm{~min}$. After $1 \mathrm{~h}$ of stirring at $-78{ }^{\circ} \mathrm{C}$, the reaction contents were quenched by the addition of saturated aqueous $\mathrm{NaHCO}_{3}(5 \mathrm{~mL})$, poured into saturated aqueous $\mathrm{NaHCO}_{3}(25 \mathrm{~mL})$, and extracted with EtOAc $(3 \square 15 \mathrm{~mL})$. The combined organic layers were then dried $\left(\mathrm{Na}_{2} \mathrm{SO}_{4}\right)$ and concentrated. The resultant residue was taken up in $\mathrm{CH}_{2} \mathrm{Cl}_{2}(4 \mathrm{~mL})$ and treated at $25^{\circ} \mathrm{C}$ with an excess of $\mathrm{NaIO}_{4}$ on silica gel. ${ }^{10}$ After $6 \mathrm{~h}$ of vigorous stirring, the reaction contents were filtered, concentrated, and purified by flash column chromatography (silica gel, hexanes/EtOAc, 1:0 4:1) to give the desired $\square$, $\square$-unsaturated ketone $(0.044 \mathrm{~g}, 88 \%$ yield $)$ as a white solid. Next, a solution of a portion of this intermediate $(0.038 \mathrm{~g}, 0.099 \mathrm{mmol}, 1.0$ equiv) in benzene $(1 \mathrm{~mL})$ was added to a solution of $\mathrm{NaOMe}(0.107 \mathrm{~g}$, $1.97 \mathrm{mmol}, 20$ equiv) and ethyl formate $(0.160 \mathrm{~mL}, 1.97 \mathrm{mmol}, 20$ equiv) in benzene $(2 \mathrm{~mL})$ at 0 ${ }^{\circ} \mathrm{C}$. After 5 min of stirring at $0{ }^{\circ} \mathrm{C}$, the resultant slurry was warmed to $25^{\circ} \mathrm{C}$ and stirred for an additional $5 \mathrm{~h}$. Upon completion, the reaction contents were quenched by the dropwise addition of saturated aqueous $\mathrm{NH}_{4} \mathrm{Cl}(1 \mathrm{~mL})$, poured into $\mathrm{H}_{2} \mathrm{O}(15 \mathrm{~mL})$, and extracted with EtOAc $(3 \square 15$ 
$\mathrm{mL})$. The combined organic layers were then washed with saturated aqueous $\mathrm{NH}_{4} \mathrm{Cl}(25 \mathrm{~mL})$ and brine $(25 \mathrm{~mL})$, dried $\left(\mathrm{MgSO}_{4}\right)$, and concentrated. The resultant yellow residue was taken up in $\mathrm{CH}_{2} \mathrm{Cl}_{2}(4 \mathrm{~mL})$, cooled to $0{ }^{\circ} \mathrm{C}$, and treated sequentially with a solution of tosyl azide ${ }^{14}(0.029 \mathrm{~g}$, $0.146 \mathrm{mmol}, 1.5$ equiv) in $\mathrm{CH}_{2} \mathrm{Cl}_{2}(1 \mathrm{~mL})$ and $\mathrm{DBU}(0.029 \mathrm{ml}, 0.194 \mathrm{mmol}, 2.0$ equiv). After 15 min of stirring at $0{ }^{\circ} \mathrm{C}$, the reaction contents were concentrated and purified directly by flash column chromatography (silica gel, hexanes/EtOAc, 1:0 4:1) to give diazoketone 27 (0.032 g, 79\% yield) as a yellow solid. 27: $\mathrm{R}_{f}=0.21$ (silica gel, hexanes/EtOAc, 9:1); m.p. = 73.5-74.5 ${ }^{\circ} \mathrm{C}$; IR (film) $\square_{\max } 2933,2867,2095,1644,1590,1466,1383,1345,1310,1169,812,756 \mathrm{~cm}^{-1}$; ${ }^{1} \mathrm{H}$ NMR $\left(400 \mathrm{MHz}, \mathrm{CDCl}_{3}\right) \square 6.89(\mathrm{~d}, J=10.4 \mathrm{~Hz}, 1 \mathrm{H}), 5.91(\mathrm{~d}, J=10.0 \mathrm{~Hz}, 1 \mathrm{H}), 2.85(\mathrm{dd}, J$ $=12.2,4.0 \mathrm{~Hz}, 1 \mathrm{H}), 2.04(\mathrm{dt}, J=12.8,3.6 \mathrm{~Hz}, 1 \mathrm{H}), 1.87-1.79(\mathrm{~m}, 2 \mathrm{H}), 1.72-1.67(\mathrm{~m}, 1 \mathrm{H})$, 1.63-1.55 (m, $3 \mathrm{H}), 1.54-1.42(\mathrm{~m}, 3 \mathrm{H}), 1.40-1.24(\mathrm{~m}, 5 \mathrm{H}), 1.21(\mathrm{dd}, J=12.8,3.6 \mathrm{~Hz}, 1 \mathrm{H})$, 1.16-1.04 (m, 7 H), 1.03 (s, 3 H), 0.98 (m, 1 H), 0.90 (d, $J=6.8 \mathrm{~Hz}, 3 \mathrm{H}), 0.85$ (dd, $J=6.8,2.0$ $\mathrm{Hz}, 6 \mathrm{H}), 0.69$ (s, $3 \mathrm{H}) ;{ }^{13} \mathrm{C}$ NMR $\left(100 \mathrm{MHz}, \mathrm{CDCl}_{3}\right) \square 184.9,152.8,127.6,64.9,56.1$, 56.0, $49.0,42.8,41.2,39.7,39.4,39.1,36.1,35.7,35.4,30.3,28.2,28.0,24.0,23.8,22.8$ (2 C), 22.5, 21.5, 18.6, 14.5, 12.1; HRMS (MALDI-FTMS) calcd for $\mathrm{C}_{27} \mathrm{H}_{43} \mathrm{~N}_{2} \mathrm{O}^{+}\left[\mathrm{M}+\mathrm{H}^{+}\right] 411.3375$, found 411.3381.

๑, -Unsaturated Ester 28. Diazoketone 27 (0.036 g, $0.088 \mathrm{mmol}, 1.0$ equiv) was dissolved in $\mathrm{MeOH}(4 \mathrm{~mL})$ and treated with $\mathrm{Et}_{3} \mathrm{~N}(0.1 \mathrm{~mL})$ at $25{ }^{\circ} \mathrm{C}$. The resultant yellow solution was degassed with bubbling nitrogen for $45 \mathrm{~min}$, and then irradiated at $25^{\circ} \mathrm{C}$ for $3 \mathrm{~h}$ using a photoreactor equipped with a $450 \mathrm{~W}$ Hanovia UV lamp (medium pressure mercury lamp) and a Pyrex cooling jacket. Once this operation was complete, the reaction contents were concentrated, taken up in DBU $(1.5 \mathrm{~mL})$, and then heated at $115^{\circ} \mathrm{C}$ for $18 \mathrm{~h}$. Upon completion, the reaction contents were purified directly by flash column chromatography (silica gel, hexanes/EtOAc, 1:0 4:1) to give the ring-contracted $\square$, $\square$-unsaturated ester $28(0.019 \mathrm{~g}, 53 \%$ yield) as a white solid. 28: $\mathrm{R}_{f}=0.51$ (silica gel, hexanes/EtOAc, 9:1); m.p. $=108-110{ }^{\circ} \mathrm{C}\left(\right.$ lit. ${ }^{15}$ m.p. $=112-113{ }^{\circ} \mathrm{C}$ ); IR (film) $\square_{\max } 2946,2869,1708,1437,1339,1243,1121,764 \mathrm{~cm}^{-1} ;{ }^{1} \mathrm{H}$ NMR (400 MHz, $\left.\mathrm{CDCl}_{3}\right) \square 6.77(\mathrm{dt}, J=5.2,3.2 \mathrm{~Hz}, 1 \mathrm{H}), 3.70$ (s, $\left.3 \mathrm{H}\right), 2.36$ (dd, $J=13.2$, 3.6 
$\mathrm{Hz}, 1 \mathrm{H}), 2.22(\mathrm{~d}, J=11.2 \mathrm{~Hz}, 1 \mathrm{H}), 2.16(\mathrm{dd}, J=16.4,3.2 \mathrm{~Hz}, 1 \mathrm{H}), 2.02-1.96(\mathrm{~m}, 2 \mathrm{H})$, $1.82-1.72(\mathrm{~m}, 3 \mathrm{H}), 1.48-0.92(\mathrm{~m}, 19 \mathrm{H}), 0.90(\mathrm{~d}, J=6.4 \mathrm{~Hz}, 3 \mathrm{H}), 0.86(\mathrm{dd}, J=6.8,2.0 \mathrm{~Hz}, 6$ $\mathrm{H}), 0.82(\mathrm{~s}, 3 \mathrm{H}), 0.66$ (s, $3 \mathrm{H}) ;{ }^{13} \mathrm{C}$ NMR (100 MHz, $\left.\mathrm{CDCl}_{3}\right) \square 166.3,144.4,138.2,56.2$ (2 C), $55.3,53.3,51.0,48.6,44.8,43.2,39.8,39.5,36.2,35.8,35.6,32.1,28.2,28.0,24.3,23.8,23.6$, 22.8, 22.5, 22.3, 18.7, 15.0, 12.3; HRMS (ESI) calcd for $\mathrm{C}_{28} \mathrm{H}_{46} \mathrm{O}_{2}^{+}\left[\mathrm{M}^{+}\right]$414.3498, found 414.3486.

Triene 29. Allyl alcohol 3 (7.0 mg, $0.024 \mathrm{mmol}, 1.0$ equiv) was taken up in THF (1.5 $\mathrm{mL})$ and treated sequentially at $25^{\circ} \mathrm{C}$ with $i$ - $\operatorname{Pr}_{2} \mathrm{NEt}(0.025 \mathrm{~mL}, 0.146 \mathrm{mmol}, 6.0$ equiv) and iodomethyl methyl ether $(0.008 \mathrm{~mL}, 0.097 \mathrm{mmol}, 4.0$ equiv). The resultant mixture was heated at $67{ }^{\circ} \mathrm{C}$ for $12 \mathrm{~h}$. Upon completion, the reaction contents were quenched by the addition of saturated aqueous $\mathrm{NH}_{4} \mathrm{Cl}(1 \mathrm{~mL})$, poured into saturated aqueous $\mathrm{NH}_{4} \mathrm{Cl}(15 \mathrm{~mL})$, and extracted with EtOAc $(3 \square 15 \mathrm{~mL})$. The combined organic layers were then dried $\left(\mathrm{MgSO}_{4}\right)$ and concentrated. The resultant light yellow oil was purified by flash column chromatography (silica gel, hexanes/EtOAc, 1:0 4:1) to give the desired MOM-protected intermediate (7.0 mg, $87 \%$

yield) as a colorless oil. Next, a two-neck, 10-mL round-bottom flask equipped with a stirring bar and a cold finger condenser was charged with $2.0 \mathrm{~mL}$ of anhydrous liquid ammonia at -78 ${ }^{\circ} \mathrm{C}$. Lithium metal $(0.010 \mathrm{~g}, 1.50 \mathrm{mmol}, 100$ equiv) was then added through the side arm of the vessel under positive argon pressure, yielding a deep blue solution upon dissolution. A portion of the MOM-protected intermediate $(5.0 \mathrm{mg}, 0.015 \mathrm{mmol}, 1.0$ equiv) was then added dropwise as a solution in THF $(1 \mathrm{~mL})$. The resultant mixture was warmed to $-40{ }^{\circ} \mathrm{C}$ and stirred for $2 \mathrm{~h}$. Upon completion, the reaction contents were treated with powdered $\mathrm{NH}_{4} \mathrm{Cl}(0.161 \mathrm{~g}, 3.00 \mathrm{mmol}$, 200 equiv); the reaction vessel was then vented with a needle and allowed to slowly warm to 0 ${ }^{\circ} \mathrm{C}$. Once this operation was complete, the residue was taken up in hexanes $/ \mathrm{H}_{2} \mathrm{O}(1: 1,5 \mathrm{ml})$, poured into $\mathrm{H}_{2} \mathrm{O}(15 \mathrm{~mL})$ and extracted with hexanes $(3 \square 15 \mathrm{~mL})$. The combined organic layers were then dried $\left(\mathrm{Na}_{2} \mathrm{SO}_{4}\right)$ and concentrated. The resultant colorless oil was purified by flash column chromatography (silica gel, hexanes) to give triene 29 (3.5 mg, 85\% yield) as a colorless oil. 29: $\mathrm{R}_{f}=0.59$ (silica gel, hexanes); $[\square]_{\mathrm{D}}{ }^{25}=-25.0^{\circ}\left(c=0.12, \mathrm{CHCl}_{3}, 90 \%\right.$ e.e.); IR (film) $\square_{\max }$ 
2958, 2921, 2850, 1648, 1447, 1385, 845, $795 \mathrm{~cm}^{-1}$; ${ }^{1} \mathrm{H}$ NMR (400 MHz, $\left.\mathrm{CDCl}_{3}\right) \square 5.20(\mathrm{~m}, 1$ H), $5.17(\mathrm{~m}, 1 \mathrm{H}), 4.86(\mathrm{~d}, J=9.6 \mathrm{~Hz}, 1 \mathrm{H}), 2.31-2.15(\mathrm{~m}, 5 \mathrm{H}), 2.15-2.02(\mathrm{~m}, 4 \mathrm{H}), 1.94(\mathrm{dd}, J$ $=16.0,2.8 \mathrm{~Hz}, 1 \mathrm{H}), 1.65(\mathrm{~m}, 2 \mathrm{H}), 1.54(\mathrm{~s}, 3 \mathrm{H}), 1.51(\mathrm{~s}, 3 \mathrm{H}), 1.42(\mathrm{~m}, 1 \mathrm{H}), 1.35(\mathrm{~m}, 1 \mathrm{H})$, $1.15(\mathrm{~s}, 3 \mathrm{H}), 1.08(\mathrm{~d}, J=7.2 \mathrm{~Hz}, 3 \mathrm{H}), 0.97(\mathrm{~d}, J=6.8 \mathrm{~Hz}, 3 \mathrm{H}) ;{ }^{13} \mathrm{C} \mathrm{NMR}\left(100 \mathrm{MHz}, \mathrm{CDCl}_{3}\right) \square$ $134.5,133.2,129.3,128.7,125.4,118.4,48.4,47.0,46.1,41.2,40.0,38.1,27.1,25.9,24.5,23.3$, 22.4, 22.2, 16.0, 15.1; HRMS (ESI) calcd for $\mathrm{C}_{20} \mathrm{H}_{32}{ }^{+}\left[\mathrm{M}^{+}\right]$272.2504, found 272.2492.

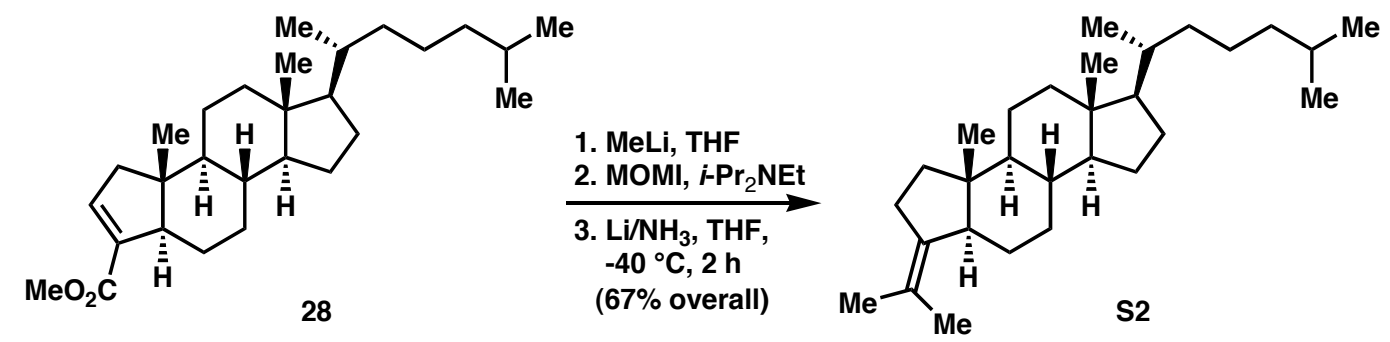

Alkene S2. Interestingly, exposure of the allyl MOM-ether derived from 28 (obtained via the same sequence described above) to similar dissolving metal reduction conditions led exclusively to $\mathbf{S 2}$, a product bearing the tetrasubstituted olefin of $\square$-araneosene (1). As such, this interesting finding reveals unique reactivity for the fused 11,5-ring system of the dolabellane diterpenoids. S2: $\mathbf{R}_{f}=0.62$ (silica gel, hexanes); IR (film) $\square_{\max } 2927,2867,1466,1457,1382$, 1264, 737, $704 \mathrm{~cm}^{-1} ;{ }^{1} \mathrm{H} \mathrm{NMR}\left(400 \mathrm{MHz} \mathrm{CDCl}_{3}\right) \square 2.24-2.08$ (m, $\left.3 \mathrm{H}\right), 1.95$ (dt, $J=12.4,3.6$ $\mathrm{Hz}, 1 \mathrm{H}), 1.87-1.74(\mathrm{~m}, 2 \mathrm{H}), 1.72(\mathrm{~m}, 1 \mathrm{H}), 1.71(\mathrm{~d}, J=1.6 \mathrm{~Hz}, 3 \mathrm{H}), 1.58(\mathrm{~d}, J=1.2 \mathrm{~Hz}, 3 \mathrm{H})$, 1.57-1.46 (m, $2 \mathrm{H}), 1.44(\mathrm{dd}, J=13.2,4.4 \mathrm{~Hz}, 1 \mathrm{H}), 1.38-1.20$ (m, $7 \mathrm{H}), 1.20-0.96$ (m, $11 \mathrm{H})$, $0.90(\mathrm{~d}, J=6.4 \mathrm{~Hz}, 3 \mathrm{H}), 0.86(\mathrm{dd}, J=6.8,2.0 \mathrm{~Hz}, 6 \mathrm{H}), 0.80(\mathrm{~m}, 1 \mathrm{H}), 0.66(\mathrm{~s}, 3 \mathrm{H}), 0.64(\mathrm{~s}, 3$ $\mathrm{H}) ;{ }^{13} \mathrm{C}$ NMR $\left(100 \mathrm{MHz}, \mathrm{CDCl}_{3}\right) \square 135.9,121.3,56.3,56.1,55.5,54.6,44.4,43.0,40.0,39.5$, $37.1,36.2,35.8,35.1,32.4,29.1,28.3,28.0,26.0,24.3,23.8,23.2,23.0,22.8,22.6,19.6,18.7$, 14.0, 12.2; HRMS (ESI) calcd for $\mathrm{C}_{29} \mathrm{H}_{50}{ }^{+}\left[\mathrm{M}^{+}\right]$398.3912, found 398.3914. 
Selected Spectra of Key Intermediates and Synthetic Natural Products

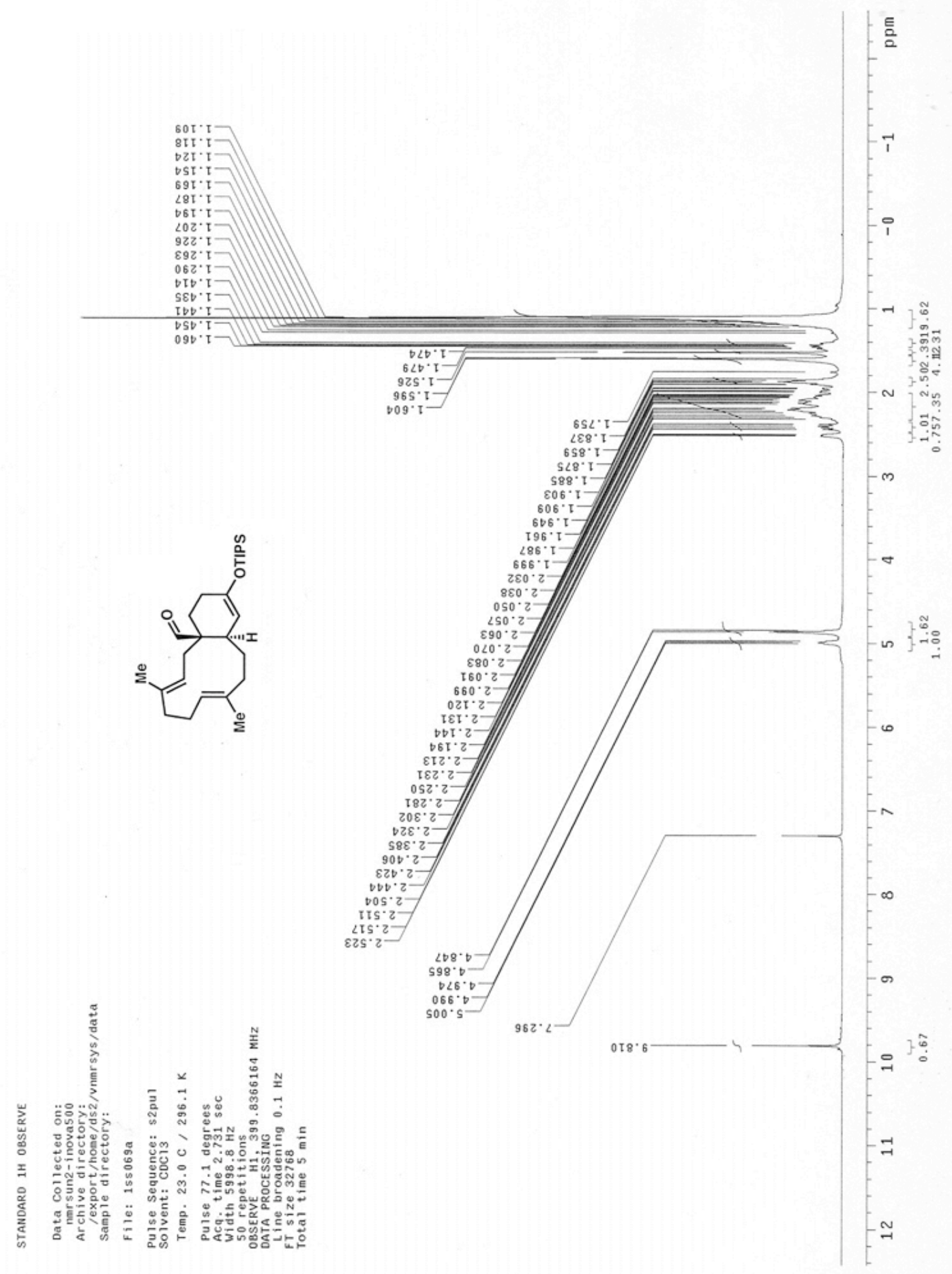




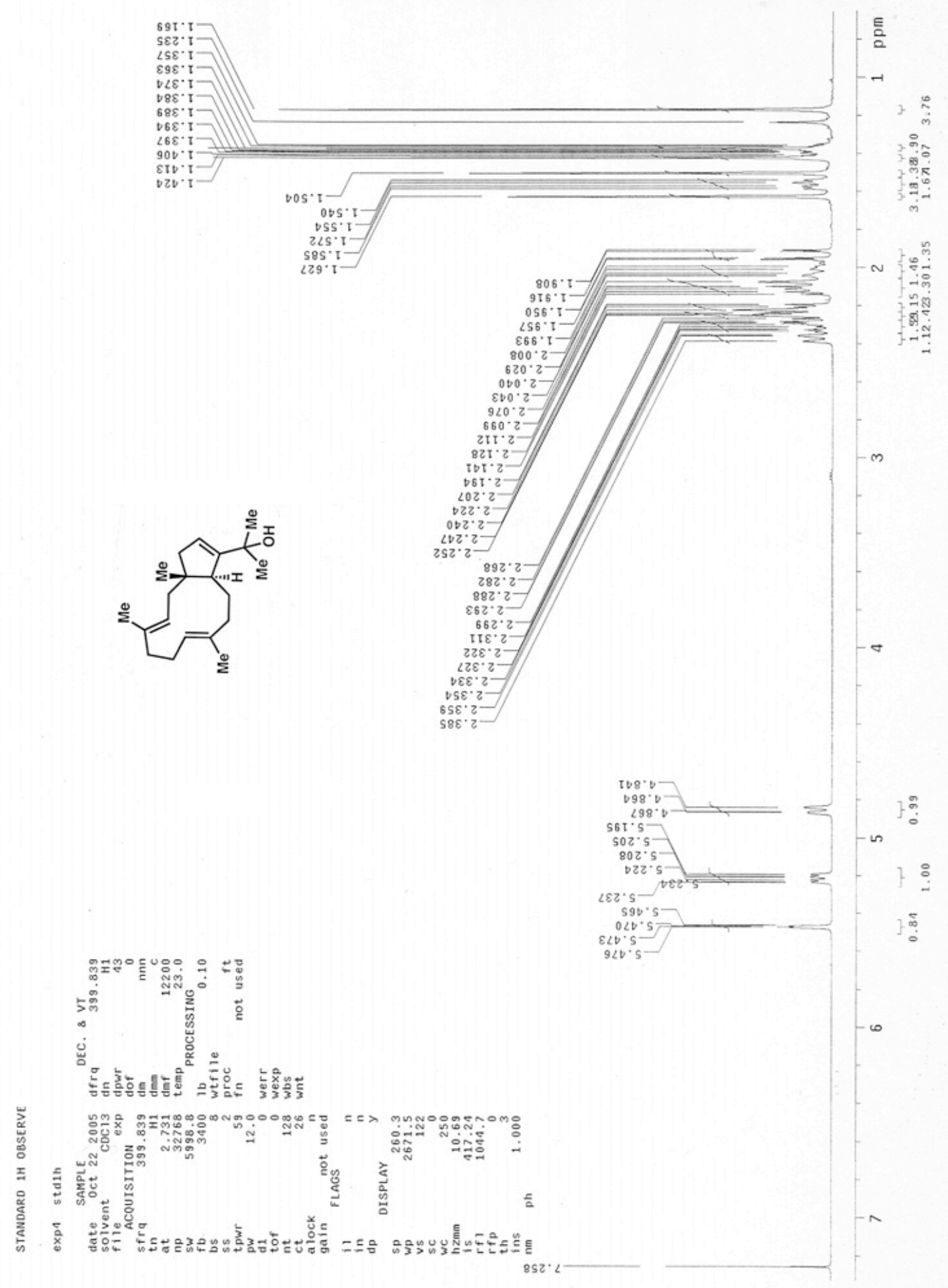




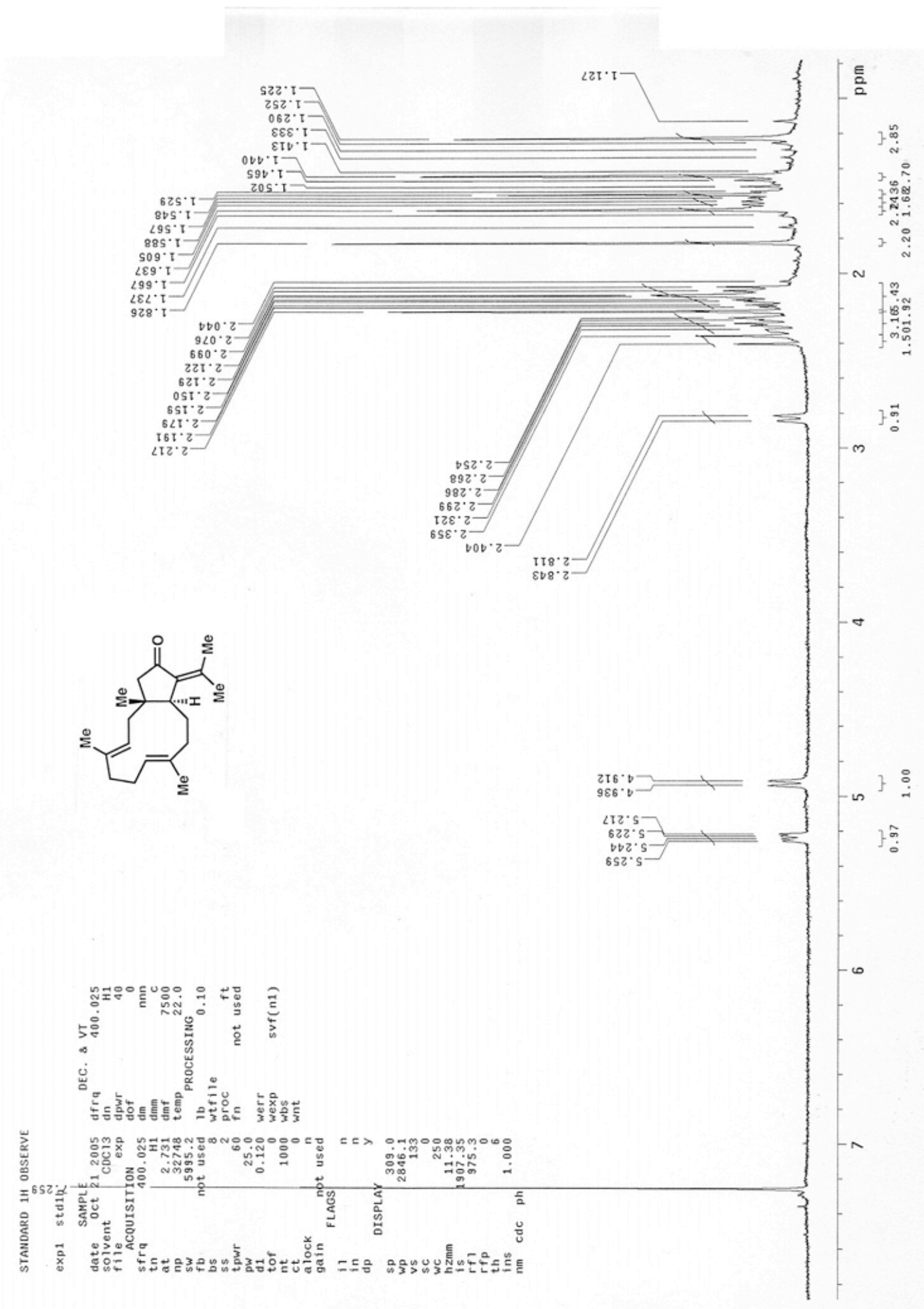




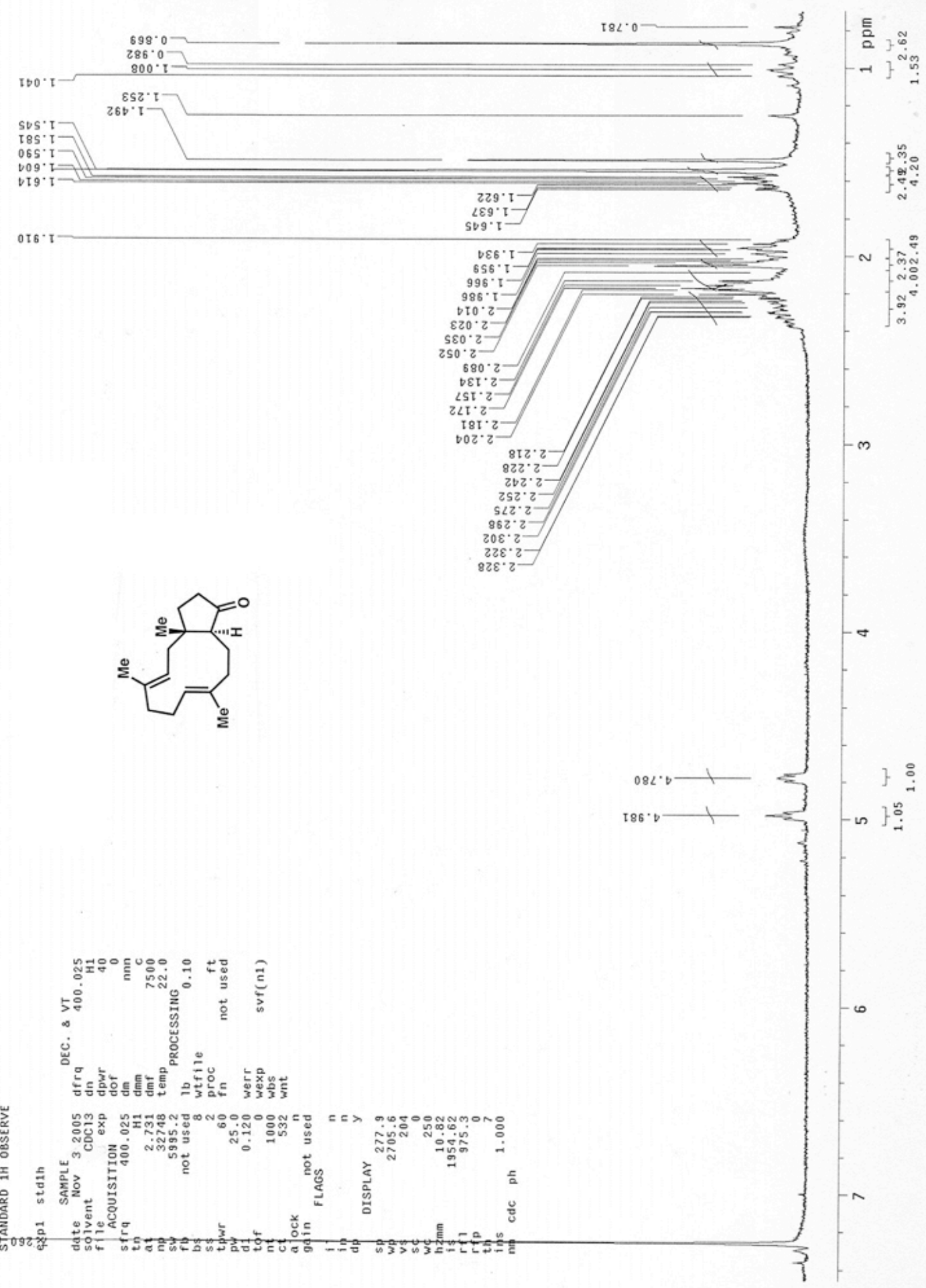




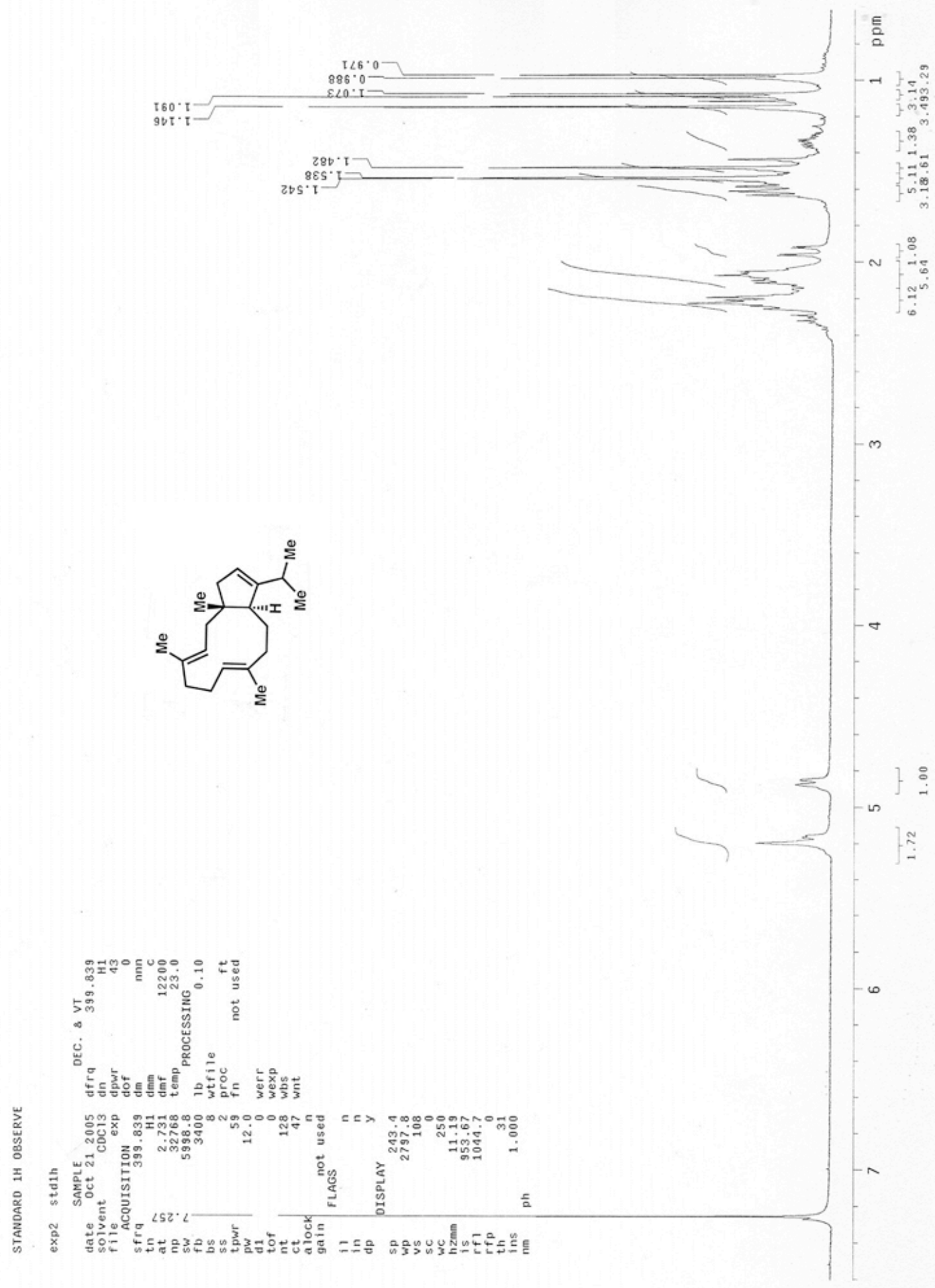




\section{References}

1. Portions of this sequence were adapted from: Zhao, Z.; Chen, H.; Li, K.; Du, W.; He, S.; Liu, H.-w. Biochemistry 2003, 42, 2089.

2. Portions of this sequence were adapted from: Corey, E. J.; Kania, R. S. J. Am. Chem. Soc. 1996, 118, 1229.

3. a) Meyer, S. D.; Schreiber, S. L. J. Org. Chem. 1994, 59, 7549. For earlier literature pertaining to this reagent, see: b) Dess, D. B.; Martin, J. C. J. Org. Chem. 1983, 48, 4155; b) Dess, D. B.; Martin, J. C. J. Am. Chem. Soc. 1991, 113, 7277.

4. Corey, E. J.; Shibata, T.; Lee, T. W. J. Am. Chem. Soc. 2002, 124, 3808.

5. Procedure adapted from: Corey, E. J.; Beames, D. J. J. Am. Chem. Soc. 1973, 95, 5829.

6. $\quad$ Frigerio, M.; Santagostino, M.; Sputore, S. J. Org. Chem. 1999, 64, 4537

7. Prepared from 2,4,6-triisopropylbenzenesulfonyl chloride and $\mathrm{NaN}_{3}$ using the procedure of: Curphey, T. J. Org. Prep. Proc. Int. 1981, 112.

8. Spectral data fully matched those previously reported: a) Shin, J.; Fenical, W. J. Org. Chem. 1991, 56, 3392; b) Rodríguez, A. D.; Acosta, A. L.; Dhasmana, H. J. Nat. Prod. 1993, 56, 1843; c) Corey, E. J.; Kania, R. S. Tetrahedron Lett. 1998, 39, 741; d) Miyaoka, H.; Isaji, Y.; Mitome, H.; Yamada, Y. Tetrahedron 2003, 59, 61.

9. Spectral data fully matched those previously reported: a) Look, S. A.; Fenical, W. J. Org. Chem. 1982, 47, 4129; b) Shin, J.; Fenical, W. J. Org. Chem. 1991, 56, 3392; c) Corey, E. J.; Kania, R. S. J. Am. Chem. Soc. 1996, 118, 1229; d) Miyaoka, H.; Isaji, Y.; Mitome, H.; Yamada, Y. Tetrahedron 2003, 59, 61.

10. Zhong, Y.-L.; Shing, T. K. M. J. Org. Chem. 1997, 62, 2622.

11. Kingsbury, J. S.; Corey, E. J. J. Am. Chem. Soc. 2005, 127, 13813.

12. Spectral data fully matched those previously reported: Xie, W.; Peng, H.; Kim, D.-I.; Kunkel, M.; Powis, G.; Zalkow, L. H. Bioorg. Med. Chem. 2001, 9, 1073.

13. The enantioselectivity of this Diels-Alder reaction was not determined.

14. Prepared from $p$-toluenesulfonyl chloride and $\mathrm{NaN}_{3}$ using the procedure of: Curphey, $\mathrm{T}$. J. Org. Prep. Proc. Int. 1981, 112.

15. Fuchs, B.; Loewenthal, H. J. E. Tetrahedron 1960, 11, 199. 